\title{
Rapidities and Observable 3-Velocities in the Flat Finslerian Event Space with Entirely Broken 3D Isotropy ${ }^{\star}$
}

George Yu. BOGOSLOVSKY

Skobeltsyn Institute of Nuclear Physics, Moscow State University, 119991 Moscow, Russia

E-mail: bogoslov@theory.sinp.msu.ru

Received December 09, 2007, in final form May 08, 2008; Published online May 26, 2008

Original article is available at http://www.emis.de/journals/SIGMA/2008/045/

\begin{abstract}
We study the geometric phase transitions that accompany the dynamic rearrangement of vacuum under spontaneous violation of initial gauge symmetry. The rearrangement may give rise to condensates of three types, namely the scalar, axially symmetric, and entirely anisotropic condensates. The flat space-time keeps being the Minkowski space in the only case of scalar condensate. The anisotropic condensate having arisen, the respective anisotropy occurs also in space-time. In this case the space-time filled with axially symmetric condensate proves to be a flat relativistically invariant Finslerian space with partially broken 3D isotropy, while the space-time filled with entirely anisotropic condensate proves to be a flat relativistically invariant Finslerian space with entirely broken 3D isotropy. The two Finslerian space types are described briefly in the extended introduction to the work, while the original part of the latter is devoted to determining observable 3 -velocities in the entirely anisotropic Finslerian event space. The main difficulties that are overcome in solving that problem arose from the nonstandard form of the light cone equation and from the necessity of correct introducing of a norm in the linear vector space of rapidities.
\end{abstract}

Key words: Lorentz, Poincaré, and gauge invariances; spontaneous symmetry breaking; dynamic rearrangement of vacuum; Finslerian space-time

2000 Mathematics Subject Classification: 53C60; 53C80; 83A05; 81T13; 81R40

\section{Introduction}

Of late years, the Lorentz symmetry violation has been widely canvassed in literature. The interest in the topic has arisen to a large extent from constructing the string-motivated phenomenological theory referred to as the Standard Model Extension (SME) [1, 2, 3].

Against the background of the research made in terms of SME, the alternative (so called Finslerian) approach to the Lorentz symmetry violation (see, in particular, $[4,5,6,7,8,9,10$, $11,12,13,14,15,16,17,18])$ gets ever more popular. The latter is based on the Finslerian, rather than pseudo-Euclidean, geometric model of flat event space. The great merit of the Finslerian model that predetermines its role in developing the fundamental interaction theory and the relativistic astrophysics arises from the fact that the model leads to Lorentz symmetry violation without violating the relativistic symmetry.

Apart from the Minkowski event space, there exist only two types of flat Finslerian spaces that exhibit relativistic symmetry, i.e. the symmetry corresponding to the Lorentz boosts. The first type Finslerian space is a space with partially broken 3D isotropy, while the second one exhibits an entirely broken 3D isotropy. Described below will be their basic properties (for more details see $[8,19])$.

\footnotetext{
*This paper is a contribution to the Proceedings of the Seventh International Conference "Symmetry in Nonlinear Mathematical Physics" (June 24-30, 2007, Kyiv, Ukraine). The full collection is available at http://www.emis.de/journals/SIGMA/symmetry2007.html
} 


\subsection{The flat relativistically-symmetric Finslerian event space with partially broken $3 \mathrm{D}$ isotropy}

The metric of the said space-time obtained in [20] is

$$
d s^{2}=\left[\frac{\left(d x_{0}-\boldsymbol{\nu} d \boldsymbol{x}\right)^{2}}{d x_{0}^{2}-d \boldsymbol{x}^{2}}\right]^{r}\left(d x_{0}^{2}-d \boldsymbol{x}^{2}\right) .
$$

This metric depends on two constant parameters, $r$ and $\boldsymbol{\nu}$, and generalizes the Minkowski metric, in which case $r$ determines the magnitude of space anisotropy, thereby characterizing the degree of deviation of metric (1) from the Minkowski metric. Instead of the 3-parameter group of rotations of Minkowski space, the given space permits only the 1-parameter group of rotations around unit vector $\boldsymbol{\nu}$ that indicates a physically preferred direction in $3 \mathrm{D}$ space. No changes occur for translational symmetry, and space-time translations leave metric (1) invariant. As to the transformations that relate the various inertial frames to each other, the ordinary Lorentz boosts modify metric (1) conformally. Therefore, they do not belong to the isometry group of space-time (1). Using them, however, we can construct invariance transformations [4] for metric (1). The corresponding generalized Lorentz transformations (generalized Lorentz boosts) prove to be

$$
x^{i}=D(\boldsymbol{v}, \boldsymbol{\nu}) R_{j}^{i}(\boldsymbol{v}, \boldsymbol{\nu}) L_{k}^{j}(\boldsymbol{v}) x^{k},
$$

where $\boldsymbol{v}$ denotes the velocities of moving (primed) inertial reference frames, the matrices $L_{k}^{j}(\boldsymbol{v})$ represent the ordinary Lorentz boosts, the matrices $R_{j}^{i}(\boldsymbol{v}, \boldsymbol{\nu})$ represent additional rotations of the spatial axes of the moving frames around the vectors $[\boldsymbol{v} \boldsymbol{\nu}]$ through the angles

$$
\varphi=\arccos \left\{1-\frac{\left(1-\sqrt{1-\boldsymbol{v}^{2} / c^{2}}\right)[\boldsymbol{v} \boldsymbol{\nu}]^{2}}{(1-\boldsymbol{v} \boldsymbol{\nu} / c) \boldsymbol{v}^{2}}\right\}
$$

of relativistic aberration of $\boldsymbol{\nu}$, and the diagonal matrices

$$
D(\boldsymbol{v}, \boldsymbol{\nu})=\left(\frac{1-\boldsymbol{v} \boldsymbol{\nu} / c}{\sqrt{1-\boldsymbol{v}^{2} / c^{2}}}\right)^{r} I
$$

stand for the additional dilatational transformations of the event coordinates.

In contrast to the ordinary Lorentz boosts, the generalized ones (2) make up a 3-parameter noncompact group with generators $X_{1}, X_{2}, X_{3}$. Thus, with the inclusion of the 1-parameter group of rotations around the preferred direction $\boldsymbol{\nu}$ and of the 4-parameter group of translations, the inhomogeneous group of isometries of event space (1) proves to have eight parameters. To obtain the simplest representation of its generators, it is sufficient to choose the third space axis along $\boldsymbol{\nu}$ and, after that, to use the infinitesimal form of transformations (2). As a result we get

$$
\begin{aligned}
& X_{1}=-\left(x^{1} p_{0}+x^{0} p_{1}\right)-\left(x^{1} p_{3}-x^{3} p_{1}\right), \quad X_{2}=-\left(x^{2} p_{0}+x^{0} p_{2}\right)+\left(x^{3} p_{2}-x^{2} p_{3}\right), \\
& X_{3}=-r x^{i} p_{i}-\left(x^{3} p_{0}+x^{0} p_{3}\right), \quad R_{3}=x^{2} p_{1}-x^{1} p_{2}, \quad p_{i}=\partial / \partial x^{i} .
\end{aligned}
$$

According to [4], the above generators satisfy the commutation relations

$$
\begin{array}{ll}
{\left[X_{1} X_{2}\right]=0,} & {\left[R_{3} X_{3}\right]=0,} \\
{\left[X_{3} X_{1}\right]=X_{1},} & {\left[R_{3} X_{1}\right]=X_{2},} \\
{\left[X_{3} X_{2}\right]=X_{2},} & {\left[R_{3} X_{2}\right]=-X_{1},} \\
{\left[p_{i} p_{j}\right]=0,} &
\end{array}
$$




$$
\begin{aligned}
& {\left[X_{1} p_{0}\right]=p_{1}, \quad\left[X_{2} p_{0}\right]=p_{2}, \quad\left[X_{3} p_{0}\right]=r p_{0}+p_{3}, \quad\left[R_{3} p_{0}\right]=0,} \\
& {\left[X_{1} p_{1}\right]=p_{0}+p_{3}, \quad\left[X_{2} p_{1}\right]=0, \quad\left[X_{3} p_{1}\right]=r p_{1}, \quad\left[R_{3} p_{1}\right]=p_{2},} \\
& {\left[X_{1} p_{2}\right]=0, \quad\left[X_{2} p_{2}\right]=p_{0}+p_{3}, \quad\left[X_{3} p_{2}\right]=r p_{2}, \quad\left[R_{3} p_{2}\right]=-p_{1},} \\
& {\left[X_{1} p_{3}\right]=-p_{1}, \quad\left[X_{2} p_{3}\right]=-p_{2}, \quad\left[X_{3} p_{3}\right]=r p_{3}+p_{0}, \quad\left[R_{3} p_{3}\right]=0 \text {. }}
\end{aligned}
$$

From this it is seen that the homogeneous isometry group of axially symmetric Finslerian event space (1) contains four parameters (generators $X_{1}, X_{2}, X_{3}$ and $R_{3}$ ). Being a subgroup of the 11-parameter similitude (Weyl) group [21], it is isomorphic to the respective 4-parameter subgroup (with generators $X_{1}, X_{2},\left.X_{3}\right|_{r=0}$ and $R_{3}$ ) of the homogeneous Lorentz group. Since the 6-parameter homogeneous Lorentz group has no 5-parameter subgroup, while the 4-parameter subgroup is unique (up to isomorphisms) [22], the transition from the Minkowski space to the axially symmetric Finslerian space (1) implies a minimum possible symmetry-breaking of the Lorentz symmetry, in which case the relativistic symmetry represented now by the generalized Lorentz boosts (2) remains preserved.

Since the light signals propagate in Finslerian event space (1) quite in the same manner as in the Minkowski space, the use [23] of the Einstein procedure of exchange of light signals makes it possible to conclude that the coordinates $x_{0}$ and $\boldsymbol{x}$ used to prescribe metric (1) have the meaning of observable Galilean coordinates of events. Accordingly, the coordinate velocity $\boldsymbol{v}=d \boldsymbol{x} / d x_{0}$ is an observable, so the Einstein law of 3-velocity addition remains valid in this case. Formally, this means that the transition from Minkowski space to Finslerian space (1) fails to alter the geometric properties of 3-velocity space; namely, the latter is again a Lobachevski space with metric

$$
d l^{2}=\frac{(d \boldsymbol{v})^{2}-[\boldsymbol{v} d \boldsymbol{v}]^{2}}{\left(1-\boldsymbol{v}^{2}\right)^{2}}
$$

with the orthogonal components of $\boldsymbol{v}$ being the Beltrami coordinates therein.

Important information about the physical displays of space anisotropy can be obtained by examining the Lagrange function [4] that corresponds to Finslerian metric (1). The function describes the peculiar non-separable interaction of a particle with a constant (i.e. $r$ and $\boldsymbol{\nu}$ being fixed) field that characterizes space anisotropy. As a result, we conclude that, despite the space anisotropy, the free inertial motion of the particle remains rectilinear and uniform as before. Contrary to the situation in the Minkowski space, however, the particle momentum direction fails to coincide with the particle velocity direction. In particular, apart from its rest energy $E=m c^{2}$, the particle has the rest momentum $\boldsymbol{p}=r m c \boldsymbol{\nu}$, while the nonrelativistic particle inert mass (that enters Newton's second law) proves to be a tensor, rather than scalar [25, 24]

$$
m_{i k}=m(1-r)\left(\delta_{i k}+r \nu_{i} \nu_{k}\right) .
$$

As to the space anisotropy impact on the behavior of fundamental fields, the correct allowance for the impact necessitates Finslerian generalization of the well-known field equations. In this case, the translational invariance of Finslerian space (1) makes the respective generalized field equations admit solutions in the form of plane waves of the type $\exp \left(i p_{k} x^{k}\right)$, where $p_{k}$ is the canonical 4-momentum (wave vector) of a particle in the anisotropic space (1), with $p_{k}$ satisfying the relativistically invariant dispersion relation [4]

$$
\left[\frac{\left(p_{0}-\boldsymbol{p} \boldsymbol{\nu}\right)^{2}}{p_{0}^{2}-\boldsymbol{p}^{2}}\right]^{-r}\left(p_{0}^{2}-\boldsymbol{p}^{2}\right)=m^{2}(1-r)^{(1-r)}(1+r)^{(1+r)} \text {. }
$$

Here and henceforth we put $c=\hbar=1$. Relation (6) permits the conclusion that the anisotropy of event space (1) in no way affects the dynamics of massless fields, electromagnetic field in 
particular. Therefore, only the fundamental massive field equations need being generalized accordingly.

If we proceed from the Klein-Gordon equation and try its generalization via the generalized dispersion relation (6) and substitution $p_{i} \rightarrow i \partial / \partial x^{i}$, we shall obtain a generalized Klein-Gordon equation in the form of either linear integral equation [25, 26] or integro-differential equation [16]. In terms of local quantum field theory, however, the Lagrangian approach to Finslerian generalization of the field equations seems to be more adequate. The initial guideline principles of constructing the respective generalized Lagrangians have been set forth in [27] and were used to demonstrate that the real massive field does not exist as a free field in Finslerian space-time (1), but does exist as a neutral component $\varphi_{2}$ of the isotopic triplet $\varphi(x)=\left\{\varphi_{1}(x), \varphi_{2}(x),-\varphi_{1}^{*}(x)\right\}$, whose generalized Lagrangian is

$$
\mathcal{L}=\varphi_{1 ; n}^{*} \varphi_{1}^{; n}+\frac{1}{2} \varphi_{2 ; n} \varphi_{2}^{; n}-\frac{m^{2}}{2}\left(1-r^{2}\right)\left[\frac{\nu^{k} j_{k}}{(1-r) m\left(2 \varphi_{1}^{*} \varphi_{1}+\varphi_{2}^{2}\right)}\right]^{\frac{2 r}{1+r}}\left(2 \varphi_{1}^{*} \varphi_{1}+\varphi_{2}^{2}\right),
$$

where $j_{k}=i\left(\varphi_{1}^{*} \varphi_{1 ; k}-\varphi_{1} \varphi_{1 ; k}^{*}\right)$.

As to the space anisotropy impact on the dynamics of massive fermion field, this effect is described by the following generalized Dirac Lagrangian [9]

$$
\mathcal{L}=\frac{i}{2}\left(\bar{\psi} \gamma^{\mu} \partial_{\mu} \psi-\partial_{\mu} \bar{\psi} \gamma^{\mu} \psi\right)-m\left[\left(\frac{\nu_{\mu} \bar{\psi} \gamma^{\mu} \psi}{\bar{\psi} \psi}\right)^{2}\right]^{r / 2} \bar{\psi} \psi
$$

where $\nu_{\mu}=(1,-\boldsymbol{\nu})$.

In contrast to the standard Dirac Lagrangian, the generalized Dirac Lagrangian leads to nonlinear spinor equations that admit a solution in the form of axially symmetric fermionantifermion condensate. The occurrence of the condensate as a physical source of the anisotropy of flat space-time (1) realizes one of the feasible mechanisms of vacuum rearrangement under spontaneous violation of the initial gauge symmetry.

Concluding the brief description of relativistically invariant Finslerian space-time (1), we cannot but mention the valuable result obtained recently in the field by G.W. Gibbons, J. Gomis and C.N. Pope. We mean the CPT operator analyzed in [16]. It is of interest to note also that, mostly, the above presented results were reproduced in [16] using the techniques of continuous deformations of the Lie algebras and nonlinear realizations. However, a different relevant notation was used in [16]. In particular, the parameter that characterizes the space anisotropy magnitude was designated $b$ instead of $r$, while the 8-parameter group of Finslerian isometries was called $\operatorname{DISIM}_{b}(2)$, i.e. Deformed Inhomogeneous SIMilitude group that includes the 2-parameter Abelian homogeneous noncompact subgroup. In our basis, the group generators and Lie algebra have the form (3) and (4), respectively. As to the finite transformations that constitute the homogeneous noncompact subgroups of $\operatorname{DISIM}_{b}(2)$, they can be found in [10].

\subsection{The flat relativistically symmetric Finslerian event space with entirely broken 3D isotropy}

The most general form of the metric of flat entirely anisotropic Finslerian event space

$$
\begin{aligned}
d s= & \left(d x_{0}-d x_{1}-d x_{2}-d x_{3}\right)^{\left(1+r_{1}+r_{2}+r_{3}\right) / 4}\left(d x_{0}-d x_{1}+d x_{2}+d x_{3}\right)^{\left(1+r_{1}-r_{2}-r_{3}\right) / 4} \\
& \times\left(d x_{0}+d x_{1}-d x_{2}+d x_{3}\right)^{\left(1-r_{1}+r_{2}-r_{3}\right) / 4}\left(d x_{0}+d x_{1}+d x_{2}-d x_{3}\right)^{\left(1-r_{1}-r_{2}+r_{3}\right) / 4}
\end{aligned}
$$

has been obtained in [19]. Three parameters $\left(r_{1}, r_{2}\right.$ and $\left.r_{3}\right)$ characterize the anisotropy of event space (8) and are restricted by the conditions

$$
1+r_{1}+r_{2}+r_{3} \geq 0, \quad 1+r_{1}-r_{2}-r_{3} \geq 0,
$$




$$
1-r_{1}+r_{2}-r_{3} \geq 0, \quad 1-r_{1}-r_{2}+r_{3} \geq 0 .
$$

It should be noted that, at $r_{1}=r_{2}=r_{3}=0$, metric (8) reduces to the fourth root of the product of four 1-forms

$$
\begin{aligned}
d s_{B-M}= & {\left[\left(d x_{0}-d x_{1}-d x_{2}-d x_{3}\right)\left(d x_{0}-d x_{1}+d x_{2}+d x_{3}\right)\right.} \\
& \left.\times\left(d x_{0}+d x_{1}-d x_{2}+d x_{3}\right)\left(d x_{0}+d x_{1}+d x_{2}-d x_{3}\right)\right]^{1 / 4} .
\end{aligned}
$$

In the given particular case, thus, we obtain the well-known Berwald-Moór metric [28, 29] written, however, in the basis introduced in [19]. As to the nonzero values of parameters $r_{i}$, the values

$$
\begin{array}{ll}
\left(r_{1}=1, r_{2}=-1, r_{3}=-1\right), & \left(r_{1}=-1, r_{2}=-1, r_{3}=1\right), \\
\left(r_{1}=-1, r_{2}=1, r_{3}=-1\right), & \left(r_{1}=1, r_{2}=1, r_{3}=1\right)
\end{array}
$$

are of particular interest. The fact is that, in case the parameters $r_{i}$ reach the said values, the metric (8), which describes the flat space-time with entirely broken 3D isotropy, degenerates into the respective 1-forms, i.e. into the total differential of absolute time:

$$
\begin{aligned}
& \left.d s\right|_{\left(r_{1}=1, r_{2}=-1, r_{3}=-1\right)}=d x_{0}-d x_{1}+d x_{2}+d x_{3}, \\
& \left.d s\right|_{\left(r_{1}=-1, r_{2}=-1, r_{3}=1\right)}=d x_{0}+d x_{1}+d x_{2}-d x_{3}, \\
& \left.d s\right|_{\left(r_{1}=-1, r_{2}=1, r_{3}=-1\right)}=d x_{0}+d x_{1}-d x_{2}+d x_{3}, \\
& \left.d s\right|_{\left(r_{1}=1, r_{2}=1, r_{3}=1\right)}=d x_{0}-d x_{1}-d x_{2}-d x_{3} .
\end{aligned}
$$

Since the same situation arises in the case of metric (1) (the latter also degenerates at $r=1$ into the total differential of absolute time), we have to conclude [19] that the absolute time is not a stable degenerate state of space-time and may turn into either partially anisotropic spacetime (1) or entirely anisotropic space-time (8). In any case, the respective geometric phase transition from the absolute time to $4 \mathrm{D}$ space-time may be treated to be an Act of Creation of $3 \mathrm{D}$ space. This phenomenon is accompanied by rearrangement of the vacuum state of the system of initially massless interacting fundamental fields, resulting in that the elementary particles acquire masses. In the case of space-time (1), the acquired particle mass is specified by tensor (5). As to space-time (8), the acquired mass is specified by the tensor

$$
m_{i k}=m\left(\begin{array}{ccc}
\left(1-r_{1}^{2}\right) & \left(r_{3}-r_{1} r_{2}\right) & \left(r_{2}-r_{1} r_{3}\right) \\
\left(r_{3}-r_{1} r_{2}\right) & \left(1-r_{2}^{2}\right) & \left(r_{1}-r_{2} r_{3}\right) \\
\left(r_{2}-r_{1} r_{3}\right) & \left(r_{1}-r_{2} r_{3}\right) & \left(1-r_{3}^{2}\right)
\end{array}\right) .
$$

Only after the above described process is complete, do the concepts of spatial extension and of reference frames become physically sensible (in a massless world, both spatial extension of anything and one or another reference frame are meaningless to speak of). It should be noted in this connection that, as early as in one of the pioneer unified gauge theories (namely, the conformal Weyl theory [30]), the very concept of space-time interval becomes physically meaningful only on violating the local conformal symmetry, resulting in that the initial massless Abelian vector gauge field acquires mass [31]. Finally attention should be paid to the fact that, formally, the absolute time serves as a connecting link, via which the correspondence principle gets satisfied for the Finslerian spaces with partially and entirely broken 3D isotropy.

Consider now the isometry group of flat Finslerian event space (8). The homogeneous 3parameter noncompact isometry group, i.e. the relativistic symmetry group of space-time (8), proves to be Abelian, while its constituent transformations have the same meaning as the conventional Lorentz boosts. The explicit form of the transformations is

$$
x_{i}^{\prime}=D L_{i k} x_{k},
$$


where

$$
D=e^{-\left(r_{1} \alpha_{1}+r_{2} \alpha_{2}+r_{3} \alpha_{3}\right)},
$$

the unimodular matrices $L_{i k}$ are

$$
\begin{aligned}
& L_{i k}=\left(\begin{array}{rrrr}
\mathcal{A} & -\mathcal{B} & -\mathcal{C} & -\mathcal{D} \\
-\mathcal{B} & \mathcal{A} & \mathcal{D} & \mathcal{C} \\
-\mathcal{C} & \mathcal{D} & \mathcal{A} & \mathcal{B} \\
-\mathcal{D} & \mathcal{C} & \mathcal{B} & \mathcal{A}
\end{array}\right), \\
& \mathcal{A}=\cosh \alpha_{1} \cosh \alpha_{2} \cosh \alpha_{3}+\sinh \alpha_{1} \sinh \alpha_{2} \sinh \alpha_{3}, \\
& \mathcal{B}=\cosh \alpha_{1} \sinh \alpha_{2} \sinh \alpha_{3}+\sinh \alpha_{1} \cosh \alpha_{2} \cosh \alpha_{3}, \\
& \mathcal{C}=\cosh \alpha_{1} \sinh \alpha_{2} \cosh \alpha_{3}+\sinh \alpha_{1} \cosh \alpha_{2} \sinh \alpha_{3}, \\
& \mathcal{D}=\cosh \alpha_{1} \cosh \alpha_{2} \sinh \alpha_{3}+\sinh \alpha_{1} \sinh \alpha_{2} \cosh \alpha_{3},
\end{aligned}
$$

with $\alpha_{1}, \alpha_{2}, \alpha_{3}$ being the group parameters. Henceforth, the coordinate velocity components $v_{i}=d x_{i} / d x_{0}$ of primed reference frame will be used as group parameters along with the parameters $\alpha_{i}$. The parameters $v_{i}$ and $\alpha_{i}$ are related to each other as

$$
\begin{aligned}
& v_{1}=\left(\tanh \alpha_{1}-\tanh \alpha_{2} \tanh \alpha_{3}\right) /\left(1-\tanh \alpha_{1} \tanh \alpha_{2} \tanh \alpha_{3}\right), \\
& v_{2}=\left(\tanh \alpha_{2}-\tanh \alpha_{1} \tanh \alpha_{3}\right) /\left(1-\tanh \alpha_{1} \tanh \alpha_{2} \tanh \alpha_{3}\right), \\
& v_{3}=\left(\tanh \alpha_{3}-\tanh \alpha_{1} \tanh \alpha_{2}\right) /\left(1-\tanh \alpha_{1} \tanh \alpha_{2} \tanh \alpha_{3}\right) .
\end{aligned}
$$

The inverse relations are

$$
\begin{aligned}
& \alpha_{1}=\frac{1}{4} \ln \frac{\left(1+v_{1}-v_{2}+v_{3}\right)\left(1+v_{1}+v_{2}-v_{3}\right)}{\left(1-v_{1}-v_{2}-v_{3}\right)\left(1-v_{1}+v_{2}+v_{3}\right)}, \\
& \alpha_{2}=\frac{1}{4} \ln \frac{\left(1-v_{1}+v_{2}+v_{3}\right)\left(1+v_{1}+v_{2}-v_{3}\right)}{\left(1-v_{1}-v_{2}-v_{3}\right)\left(1+v_{1}-v_{2}+v_{3}\right)}, \\
& \alpha_{3}=\frac{1}{4} \ln \frac{\left(1-v_{1}+v_{2}+v_{3}\right)\left(1+v_{1}-v_{2}+v_{3}\right)}{\left(1-v_{1}-v_{2}-v_{3}\right)\left(1+v_{1}+v_{2}-v_{3}\right)} .
\end{aligned}
$$

As to generators $X_{i}$ of homogeneous 3-parameter isometry group (10) of space-time (8), they can be presented as

$$
\begin{aligned}
& X_{1}=-r_{1} x_{\alpha} p_{\alpha}-\left(x_{1} p_{0}+x_{0} p_{1}\right)+\left(x_{2} p_{3}+x_{3} p_{2}\right), \\
& X_{2}=-r_{2} x_{\alpha} p_{\alpha}-\left(x_{2} p_{0}+x_{0} p_{2}\right)+\left(x_{1} p_{3}+x_{3} p_{1}\right), \\
& X_{3}=-r_{3} x_{\alpha} p_{\alpha}-\left(x_{3} p_{0}+x_{0} p_{3}\right)+\left(x_{1} p_{2}+x_{2} p_{1}\right),
\end{aligned}
$$

where $p_{\alpha}=\partial / \partial x_{\alpha}$ are generators of the 4-parameter group of translations. Thus, on including the latter, the inhomogeneous group of isometries of entirely anisotropic Finslerian event space (8) turns out to be a 7-parameter group. As to its generators, they satisfy the commutation relations

$$
\begin{aligned}
& {\left[X_{i} X_{j}\right]=0, \quad\left[p_{\alpha} p_{\beta}\right]=0,} \\
& {\left[X_{1} p_{0}\right]=r_{1} p_{0}+p_{1}, \quad\left[X_{2} p_{0}\right]=r_{2} p_{0}+p_{2}, \quad\left[X_{3} p_{0}\right]=r_{3} p_{0}+p_{3},} \\
& {\left[X_{1} p_{1}\right]=r_{1} p_{1}+p_{0}, \quad\left[X_{2} p_{1}\right]=r_{2} p_{1}-p_{3}, \quad\left[X_{3} p_{1}\right]=r_{3} p_{1}-p_{2},} \\
& {\left[X_{1} p_{2}\right]=r_{1} p_{2}-p_{3}, \quad\left[X_{2} p_{2}\right]=r_{2} p_{2}+p_{0}, \quad\left[X_{3} p_{2}\right]=r_{3} p_{2}-p_{1},} \\
& {\left[X_{1} p_{3}\right]=r_{1} p_{3}-p_{2}, \quad\left[X_{2} p_{3}\right]=r_{2} p_{3}-p_{1}, \quad\left[X_{3} p_{3}\right]=r_{3} p_{3}+p_{0} \text {. }}
\end{aligned}
$$


Concluding the introductory part of the work, we wish to note that the next (original) part continues studying the flat relativistically invariant Finslerian event space with entirely broken 3D isotropy (expression (8)). The point is that, contrary to metric (1), the coordinates $x_{0}, x_{i}$ used to prescribe metric (8) are not the orthogonal Galilean coordinates of events. Accordingly, the 3 -velocity $v_{i}=d x_{i} / d x_{0}$ is not an observable, but is only meaningful as being a coordinate 3 velocity in event space (8). It should be noted apropos that, within the framework of conventional special relativity, the coordinate 3 -velocity that corresponds to nonorthogonal coordinates is not an observable 3-velocity either and, to determine the latter, there exists the well-known algorithm. From this example alone it follows that physical identification of various quantities and relations arising in terms of the model for flat Finslerian space of events (8) deserves special attention and turns sometimes into an independent problem. One of the like problems that permits a novel approach to interpreting some astrophysical observations, in particular the data relevant to the temperature anisotropy of the microwave background radiation, will be solved below. We mean the problem of determining observable 3 -velocities within the framework of the model for entirely anisotropic Finslerian event space (8). An appropriate algorithm that, in particular, permits the magnitude of observable 3 -velocity to be expressed via the components of the latter must start being constructed by considering the space of coordinate 3 -velocities.

\section{The components of relative coordinate velocity of two particles}

Obviously, the group of generalized Lorentz boosts (10) that acts in entirely anisotropic event space (8) induces the group of the respective transformations in the space of coordinate 3velocities $v_{i}=d x_{i} / d x_{0}$. To obtain the transformations, let equations (10) be rewritten in terms of coordinate differentials

$$
\begin{aligned}
& d x_{0}^{\prime}=D\left(\mathcal{A} d x_{0}-\mathcal{B} d x_{1}-\mathcal{C} d x_{2}-\mathcal{D} d x_{3}\right), \\
& d x_{1}^{\prime}=D\left(-\mathcal{B} d x_{0}+\mathcal{A} d x_{1}+\mathcal{D} d x_{2}+\mathcal{C} d x_{3}\right), \\
& d x_{2}^{\prime}=D\left(-\mathcal{C} d x_{0}+\mathcal{D} d x_{1}+\mathcal{A} d x_{2}+\mathcal{B} d x_{3}\right), \\
& d x_{3}^{\prime}=D\left(-\mathcal{D} d x_{0}+\mathcal{C} d x_{1}+\mathcal{B} d x_{2}+\mathcal{A} d x_{3}\right) .
\end{aligned}
$$

After dividing, then, the second, third, and fourth relations by the first one and using the fact that $v_{i}=d x_{i} / d x_{0}$ are components of the coordinate 3 -velocity, we get

$$
\begin{aligned}
v_{1}^{\prime} & =\frac{-\mathcal{B}+\mathcal{A} v_{1}+\mathcal{D} v_{2}+\mathcal{C} v_{3}}{\mathcal{A}-\mathcal{B} v_{1}-\mathcal{C} v_{2}-\mathcal{D} v_{3}}, \quad v_{2}^{\prime}=\frac{-\mathcal{C}+\mathcal{D} v_{1}+\mathcal{A} v_{2}+\mathcal{B} v_{3}}{\mathcal{A}-\mathcal{B} v_{1}-\mathcal{C} v_{2}-\mathcal{D} v_{3}}, \\
v_{3}^{\prime} & =\frac{-\mathcal{D}+\mathcal{C} v_{1}+\mathcal{B} v_{2}+\mathcal{A} v_{3}}{\mathcal{A}-\mathcal{B} v_{1}-\mathcal{C} v_{2}-\mathcal{D} v_{3}}
\end{aligned}
$$

The relations (14) interrelate the components $v_{i}$ and $v_{i}^{\prime}$ of the coordinate 3 -velocity of a particle in the initial and primed inertial frames, respectively, with the dependence of the involved matrix elements $\mathcal{A}, \mathcal{B}, \mathcal{C}, \mathcal{D}$ on the coordinate 3 -velocity of the primed reference frame being determined by relations (11) and (13). Formally, the transformations (14) are a nonlinear representation of linear group (10). Besides, it should be noted that the representation proves to be independent of parameters $r_{i}$ and, therefore, is equally valid for the Berwald-Moór metric.

Consider now two particles. Let $\stackrel{(2)}{v_{i}}$ be the coordinate 3 -velocity of the second particle in the initial reference frame, and $\stackrel{(1)}{v}_{i}$ be the coordinate 3 -velocity of the first particle in the same frame. In addition, let $\stackrel{(1)}{v_{i}}$ be identified as the coordinate 3 -velocity of primed reference frame. As a result, the primed frame acquires the meaning of the rest frame of the first particle. Finally, 
let the 3-velocity components of the second particle in the rest frame of the first particle (or, in other words, the relative velocity of the second particle with respect to the first particle) be

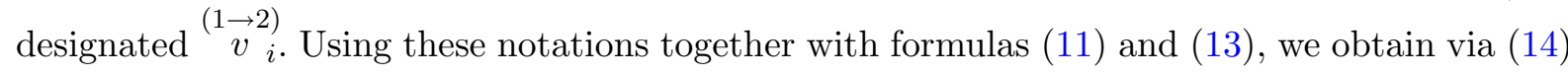

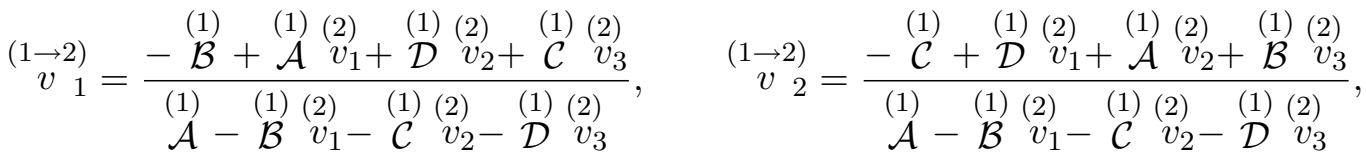

$$
\begin{aligned}
& \stackrel{(1 \rightarrow}{v}_{3}^{2)}=\frac{-\stackrel{(1)}{\mathcal{D}}+\stackrel{(1)}{\mathcal{C}} \stackrel{(2)}{v}_{1}+\stackrel{(1)}{\mathcal{B}} \stackrel{(2)}{v}_{2}+\stackrel{(1)}{\mathcal{A}} \stackrel{(2)}{v}_{3}}{\stackrel{(1)}{\mathcal{A}}-\stackrel{(1)}{\mathcal{B}} \stackrel{(2)}{v}_{1}-\stackrel{(1)}{\mathcal{C}} \stackrel{(2)}{v}_{2}-\stackrel{(1)}{\mathcal{D}} \stackrel{(2)}{v}_{3}}
\end{aligned}
$$

where

$$
\begin{aligned}
& \stackrel{(1)}{\mathcal{A}}=\frac{1-\stackrel{(1)}{v_{1}} 2-\stackrel{(1)}{v_{2}} 2-\stackrel{(1)}{v_{3}} 2-2 \stackrel{(1)}{v_{1}} \stackrel{(1)}{v}_{2} \stackrel{(1)}{v}_{3}}{\left[\left(1-\stackrel{(1)}{v_{1}}-\stackrel{(1)}{v_{2}}-\stackrel{(1)}{v_{3}}\right)\left(1-\stackrel{(1)}{v_{1}}+\stackrel{(1)}{v_{2}}+\stackrel{(1)}{v_{3}}\right)\left(1+\stackrel{(1)}{v_{1}}-\stackrel{(1)}{v_{2}}+\stackrel{(1)}{v_{3}}\right)\left(1+\stackrel{(1)}{v_{1}}+\stackrel{(1)}{v_{2}}-\stackrel{(1)}{v_{3}}\right)\right]^{3 / 4}}, \\
& \stackrel{(1)}{\mathcal{B}}=\frac{\left(\stackrel{1)}{v_{1}}+2 \stackrel{(1)}{v_{2}} \stackrel{(1)}{v_{3}}-\stackrel{(1)}{v_{1}}\left(\stackrel{(1)}{v}_{1}^{2}-\stackrel{(1)}{v_{2}}-\stackrel{(1)}{v_{3}}\right)\right.}{\left[\left(1-\stackrel{(1)}{v_{1}}-\stackrel{(1)}{v_{2}}-\stackrel{(1)}{v_{3}}\right)\left(1-\stackrel{(1)}{v_{1}}+\stackrel{(1)}{v_{2}}+\stackrel{(1)}{v_{3}}\right)\left(1+\stackrel{(1)}{v_{1}}-\stackrel{(1)}{v_{2}}+\stackrel{(1)}{v_{3}}\right)\left(1+\stackrel{(1)}{v_{1}}+\stackrel{(1)}{v_{2}}-\stackrel{(1)}{v_{3}}\right)\right]^{3 / 4}}, \\
& \stackrel{(1)}{\mathcal{C}}=\frac{\left(\stackrel{1)}{v_{2}}+2 \stackrel{(1)}{v_{1}} \stackrel{(1)}{v_{3}}-\stackrel{(1)}{v_{2}}\left(\stackrel{(1)}{v}_{2}^{2}-\stackrel{(1)}{v_{1}}-\stackrel{(1)}{v_{3}}\right)\right.}{\left[\left(1-\stackrel{(1)}{v_{1}}-\stackrel{(1)}{v_{2}}-\stackrel{(1)}{v_{3}}\right)\left(1-\stackrel{(1)}{v_{1}}+\stackrel{(1)}{v_{2}}+\stackrel{(1)}{v_{3}}\right)\left(1+\stackrel{(1)}{v_{1}}-\stackrel{(1)}{v_{2}}+\stackrel{(1)}{v_{3}}\right)\left(1+\stackrel{(1)}{v_{1}}+\stackrel{(1)}{v_{2}}-\stackrel{(1)}{v_{3}}\right)\right]^{3 / 4}},
\end{aligned}
$$

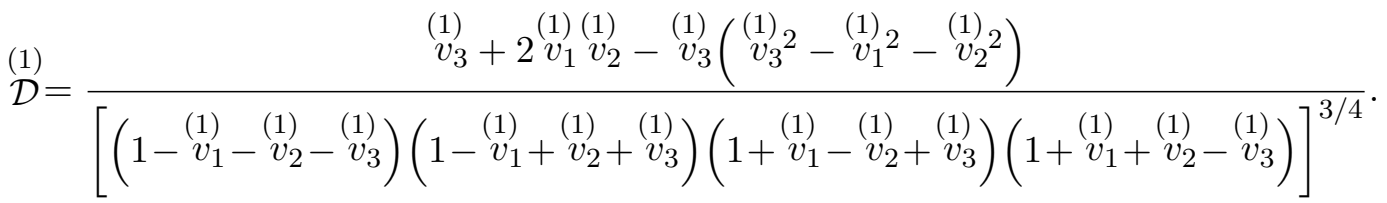

The above cumbersome formulas express the components of the relative coordinate 3-velocity of two particles via the coordinate 3-velocity components of either particle. From the fact that the above relations are a direct consequence of transformations (10) that constitute the Abelian group with parameters $\alpha_{i}$, while $\alpha_{i}$ proper can be treated to be rapidity components related to $v_{i}$ via (12) and (13), the conclusion inevitably comes to mind that, in terms of $\alpha_{i}$, relations (15) must get simplified significantly and take on the form

$$
\stackrel{(1 \rightarrow 2)}{\alpha}_{i}=\stackrel{(2)}{\alpha}_{i}-\stackrel{(1)}{\alpha}_{i}
$$

The fact that the conclusion is really true can be proved as follows. First, proceeding from (15), and via direct calculations we get the following three equations

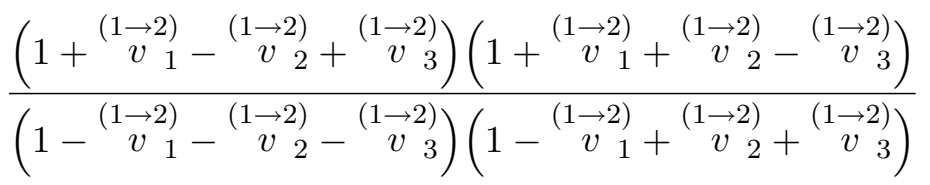

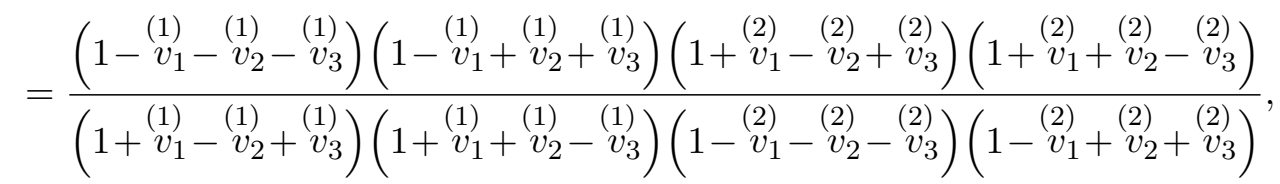




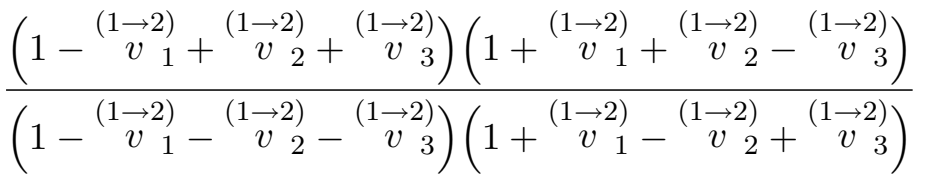

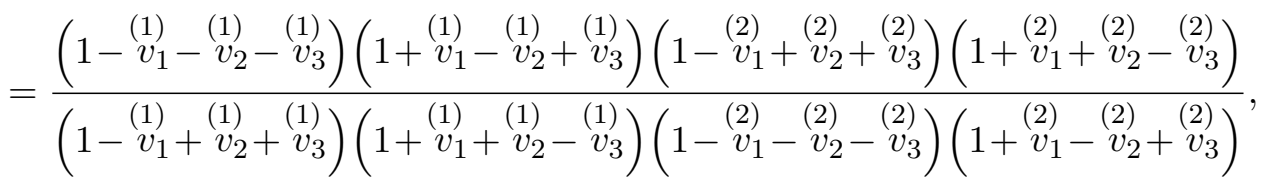

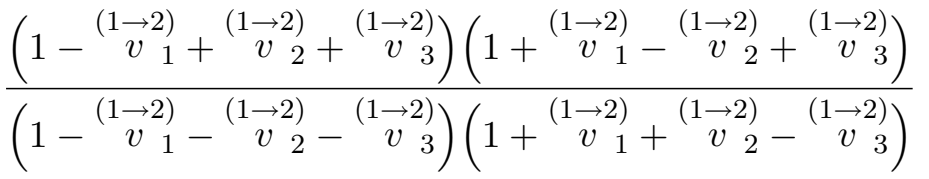

$$
\begin{aligned}
& =\frac{\left(1-\stackrel{(1)}{v_{1}}-\stackrel{(1)}{v_{2}}-\stackrel{(1)}{v_{3}}\right)\left(1+\stackrel{(1)}{v_{1}}+\stackrel{(1)}{v_{2}}-\stackrel{(1)}{v_{3}}\right)\left(1-\stackrel{(2)}{v_{1}}+\stackrel{(2)}{v_{2}}+\stackrel{(2)}{v_{3}}\right)\left(1+\stackrel{(2)}{v_{1}}-\stackrel{(2)}{v_{2}}+\stackrel{(2)}{v_{3}}\right)}{\left(1-\stackrel{(1)}{v}_{1}+\stackrel{(1)}{v_{2}}+\stackrel{(1)}{v_{3}}\right)\left(1+\stackrel{(1)}{v_{1}}-\stackrel{(1)}{v_{2}}+\stackrel{(1)}{v_{3}}\right)\left(1-\stackrel{(2)}{v_{1}}-\stackrel{(2)}{v_{2}}-\stackrel{(2)}{v_{3}}\right)\left(1+\stackrel{(2)}{v_{1}}+\stackrel{(2)}{v_{2}}-\stackrel{(2)}{v_{3}}\right)},
\end{aligned}
$$

whereupon we are only to find natural logarithm of each of the equations and use formulas (13). The result thereof is just relation (16).

According to (16), the rapidities $\alpha_{i}$ form the linear vector space, whence the problem of introducing of a norm $\left|\alpha_{i}\right|$ in that vector space arises for us to solve.

\section{Introducing of a norm in the vector space of rapidities}

To find $\left|\alpha_{i}\right|$ as an explicit function of $\alpha_{i}$, attention should be paid to relation

$$
\stackrel{(1 \rightarrow 2)}{\alpha}_{i}=-{ }^{(2 \rightarrow 1)}{ }_{i},
$$

which means that the rapidity components of the second particle relative to the first particle differ in sign from the rapidity components of the first particle relative to the second. This fact is a trivial consequence of the Abelian structure of relativistic symmetry group (10) and prompts us to write $\left|\alpha_{i}\right|=\sqrt{\alpha_{1}^{2}+\alpha_{2}^{2}+\alpha_{3}^{2}}$. However, such introducing of a norm in the vector space of rapidities would be physically incorrect because it would never lead to any reasonable relation between the components of observable 3 -velocity and its magnitude. Therefore, we will be acting as follows.

First, proceeding from physical considerations, we shall express the square of observable 3velocity $V^{2}$ to be an explicit function of the components of coordinate 3 -velocity $v_{i}$. After that, formulas (12) are to be used to represent $V^{2}$ as an explicit function of the rapidity components $\alpha_{i}$. Thereby, we shall actually finish the procedure of introducing of the norm since (by definition) the magnitude $V$ of observable 3-velocity and the rapidity magnitude $\left|\alpha_{i}\right|$ are related to one another as

$$
V^{2}=\tanh ^{2}\left|\alpha_{i}\right|
$$

Thus, let us, first of all, find the explicit form of function $V^{2}=V^{2}\left(v_{i}\right)$. In accordance with its physical meaning, that function, just as $V\left(v_{i}\right)$, must satisfy the condition

$$
V^{2}\left(\begin{array}{c}
\left(\vec{v}_{i}^{2}\right) \\
\end{array}\right)=V^{2}\left(\begin{array}{c}
(2 \rightarrow 1) \\
v
\end{array}\right)
$$

where $\stackrel{(1 \rightarrow 2)}{v}$ are the components of coordinate 3 -velocity of the second particle with respect to the first one, and $\stackrel{(2 \rightarrow 1)}{v}_{i}$ are the components of coordinate 3 -velocity of the first particle with 
respect to the second one. Considering $v_{i}$ as the group parameters that are alternative to the parameters $\alpha_{i}$, we can see that $\stackrel{(1 \rightarrow 2)}{v}_{i}$ and $\stackrel{(2 \rightarrow 1)}{v}_{i}$ are the mutually inverse elements $g$ and $g^{-1}$ of group (10). In this case, in terms of $\alpha_{i}$, the transition $g \rightarrow g^{-1}$ corresponds (see formula (17)) to transformation $\alpha_{i} \rightarrow \tilde{\alpha}_{i}=-\alpha_{i}$, whereas in terms of $v_{i}$, the same transition corresponds (in virtue of (12) and (13)) to transformation

$$
\begin{aligned}
& v_{1} \rightarrow \tilde{v}_{1}=-\frac{v_{1}\left(1-v_{1}^{2}+v_{2}^{2}+v_{3}^{2}\right)+2 v_{2} v_{3}}{1-v_{1}^{2}-v_{2}^{2}-v_{3}^{2}-2 v_{1} v_{2} v_{3}}, \quad v_{2} \rightarrow \tilde{v}_{2}=-\frac{v_{2}\left(1+v_{1}^{2}-v_{2}^{2}+v_{3}^{2}\right)+2 v_{1} v_{3}}{1-v_{1}^{2}-v_{2}^{2}-v_{3}^{2}-2 v_{1} v_{2} v_{3}}, \\
& v_{3} \rightarrow \tilde{v}_{3}=-\frac{v_{3}\left(1+v_{1}^{2}+v_{2}^{2}-v_{3}^{2}\right)+2 v_{1} v_{2}}{1-v_{1}^{2}-v_{2}^{2}-v_{3}^{2}-2 v_{1} v_{2} v_{3}}
\end{aligned}
$$

Accordingly, the inverse transformations appear as $-\alpha_{i}=\tilde{\alpha}_{i} \rightarrow \alpha_{i}$ and

$$
\begin{aligned}
& \tilde{v}_{1} \rightarrow v_{1}=-\frac{\tilde{v}_{1}\left(1-\tilde{v}_{1}^{2}+\tilde{v}_{2}^{2}+\tilde{v}_{3}^{2}\right)+2 \tilde{v}_{2} \tilde{v}_{3}}{1-\tilde{v}_{1}^{2}-\tilde{v}_{2}^{2}-\tilde{v}_{3}^{2}-2 \tilde{v}_{1} \tilde{v}_{2} \tilde{v}_{3}}, \quad \tilde{v}_{2} \rightarrow v_{2}=-\frac{\tilde{v}_{2}\left(1+\tilde{v}_{1}^{2}-\tilde{v}_{2}^{2}+\tilde{v}_{3}^{2}\right)+2 \tilde{v}_{1} \tilde{v}_{3}}{1-\tilde{v}_{1}^{2}-\tilde{v}_{2}^{2}-\tilde{v}_{3}^{2}-2 \tilde{v}_{1} \tilde{v}_{2} \tilde{v}_{3}}, \\
& \tilde{v}_{3} \rightarrow v_{3}=-\frac{\tilde{v}_{3}\left(1+\tilde{v}_{1}^{2}+\tilde{v}_{2}^{2}-\tilde{v}_{3}^{2}\right)+2 \tilde{v}_{1} \tilde{v}_{2}}{1-\tilde{v}_{1}^{2}-\tilde{v}_{2}^{2}-\tilde{v}_{3}^{2}-2 \tilde{v}_{1} \tilde{v}_{2} \tilde{v}_{3}} .
\end{aligned}
$$

Now we see that equation (19), i.e. the first, and most important, restriction on the sought function $V^{2}$, can be rewritten as

$$
V^{2}\left(v_{i}\right)=V^{2}\left(\tilde{v}_{i}\right)
$$

Thus, the function $V^{2}\left(v_{i}\right)$ must be an invariant of transformations (20) and (21).

Since, when examining a classical particle motion, we deal with causally-related events, the physically permissible range of the values of squared observable 3 -velocity is restricted by the condition $0 \leq V^{2}\left(v_{i}\right) \leq 1$, where

$$
V^{2}(0,0,0)=0
$$

in accordance with the definition of coordinate 3 -velocity $v_{i}$. As to the range of physically permissible $v_{i}$ values, that range will be shown in the next section to be restricted by the regular tetrahedron surface presented in Fig. 6. On the tetrahedron surface proper, the coordinate 3velocities, which coincide with all the possible coordinate 3 -velocities of a photon, satisfy the relation

$$
\left(d s_{B-M} / d x_{0}\right)^{4}=\left(1-v_{1}-v_{2}-v_{3}\right)\left(1-v_{1}+v_{2}+v_{3}\right)\left(1+v_{1}-v_{2}+v_{3}\right)\left(1+v_{1}+v_{2}-v_{3}\right)=0 .
$$

Since, on the other hand, $V^{2}=1$ at any direction of observable 3 -velocity of the photon, relation (24) implies that the sought function $V^{2}\left(v_{i}\right)$ must satisfy the condition

$$
\left.V^{2}\right|_{\left(d s_{B-M} / d x_{0}=0\right)}=1 \text {. }
$$

Apart from the stated conditions (22), (23), and (25), attention should be paid to the fact that, in the $2 \mathrm{D}$ case, where (for instance) $d x_{0} \neq 0, d x_{1} \neq 0, d x_{2}=d x_{3}=0$, the Berwald-Moór space coincides with the Minkowski space, i.e. $d s_{B-M}^{2}=d x_{0}^{2}-d x_{1}^{2}$. In this case, accordingly, the coordinate velocity $v_{1}$ coincides with the observable velocity $V$. As a result, we conclude that the sought function $V^{2}\left(v_{i}\right)$ must also satisfy the relations

$$
V^{2}\left(v_{1}, 0,0\right)=v_{1}^{2}, \quad V^{2}\left(0, v_{2}, 0\right)=v_{2}^{2}, \quad V^{2}\left(0,0, v_{3}\right)=v_{3}^{2} .
$$

To find the function $V^{2}\left(v_{i}\right)$ that satisfies the conditions (22), (23), (25), and (26), an attempt will be made firstly to construct some auxiliary function $f\left(v_{i}\right)$ that would remain invariant under 


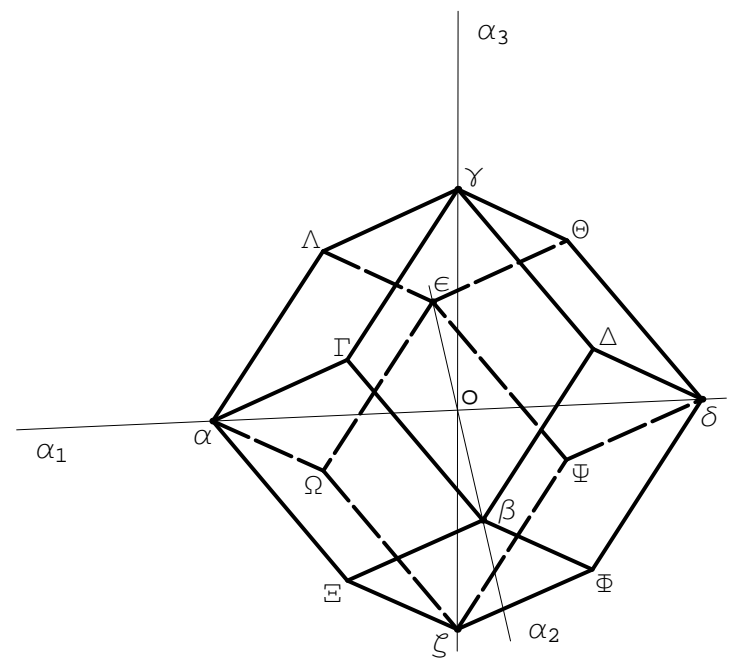

Figure 1. The regular rhombic dodecahedron in the space of rapidities $\alpha_{i}$ as an image (at $\tanh \left|\alpha_{i}\right|=1$ ) of the light front surface.

transformations (20) and (21). To that end, consider two characteristic functions that enter relations (20) and (24) above, namely

$$
f_{1}\left(v_{i}\right)=1-v_{1}^{2}-v_{2}^{2}-v_{3}^{2}-2 v_{1} v_{2} v_{3}
$$

and

$$
f_{2}\left(v_{i}\right)=\left(1-v_{1}-v_{2}-v_{3}\right)\left(1-v_{1}+v_{2}+v_{3}\right)\left(1+v_{1}-v_{2}+v_{3}\right)\left(1+v_{1}+v_{2}-v_{3}\right) .
$$

Using substitution (20), we can readily prove that $f_{1}\left(\tilde{v}_{i}\right)=f_{2}^{2}\left(v_{i}\right) / f_{1}^{3}\left(v_{i}\right)$. Similarly, we can verify that $f_{2}\left(\tilde{v}_{i}\right)=f_{2}^{3}\left(v_{i}\right) / f_{1}^{4}\left(v_{i}\right)$. Therefore, on determining the function $f\left(v_{i}\right)$ via relation $f\left(v_{i}\right)=f_{2}\left(v_{i}\right) / f_{1}\left(v_{i}\right)$, we come to equality $f\left(\tilde{v}_{i}\right)=f\left(v_{i}\right)$, which means that the function $f\left(v_{i}\right)$ introduced as above is actually an invariant of transformations (20) and (21). From this it follows clearly that the sought function $V^{2}\left(v_{i}\right)$, which satisfies the conditions (22), (23), (25), and (26), is prescribed by the relation $V^{2}\left(v_{i}\right)=1-f\left(v_{i}\right)=1-f_{2}\left(v_{i}\right) / f_{1}\left(v_{i}\right)$ and (owing to (27) and $(28))$ proves to be

$$
V^{2}\left(v_{i}\right)=1-\frac{\left(1-v_{1}-v_{2}-v_{3}\right)\left(1-v_{1}+v_{2}+v_{3}\right)\left(1+v_{1}-v_{2}+v_{3}\right)\left(1+v_{1}+v_{2}-v_{3}\right)}{1-v_{1}^{2}-v_{2}^{2}-v_{3}^{2}-2 v_{1} v_{2} v_{3}} .
$$

Now, representing (via (29) and (12)) $V^{2}$ as an explicit function of $\alpha_{i}$ and using definition (18), we get finally

$$
\tanh ^{2}\left|\alpha_{i}\right|=\frac{\tanh ^{2} \alpha_{1}\left(1-\tanh ^{2} \alpha_{2}\right)+\tanh ^{2} \alpha_{2}\left(1-\tanh ^{2} \alpha_{3}\right)+\tanh ^{2} \alpha_{3}\left(1-\tanh ^{2} \alpha_{1}\right)}{1-\tanh ^{2} \alpha_{1} \tanh ^{2} \alpha_{2} \tanh ^{2} \alpha_{3}} .
$$

This formula expresses the rapidity magnitude $\left|\alpha_{i}\right|$ via rapidity components $\alpha_{i}$. As should be in this case, $\left|\alpha_{i}\right|$ is a convex function of its own arguments $\alpha_{i}$, which is invariant with respect to their reflection $\alpha_{i} \leftrightarrow-\alpha_{i}$. According to (30),

$$
\left|\alpha_{i}\right| \approx \sqrt{\alpha_{1}^{2}+\alpha_{2}^{2}+\alpha_{3}^{2}}
$$

at small $\left|\alpha_{i}\right|$ values. As $\left|\alpha_{i}\right|$ increases, the spheres (31) get deformed and transformed at $\left|\alpha_{i}\right| \rightarrow \infty$ into the regular rhombic dodecahedron shown in Fig. 1. 

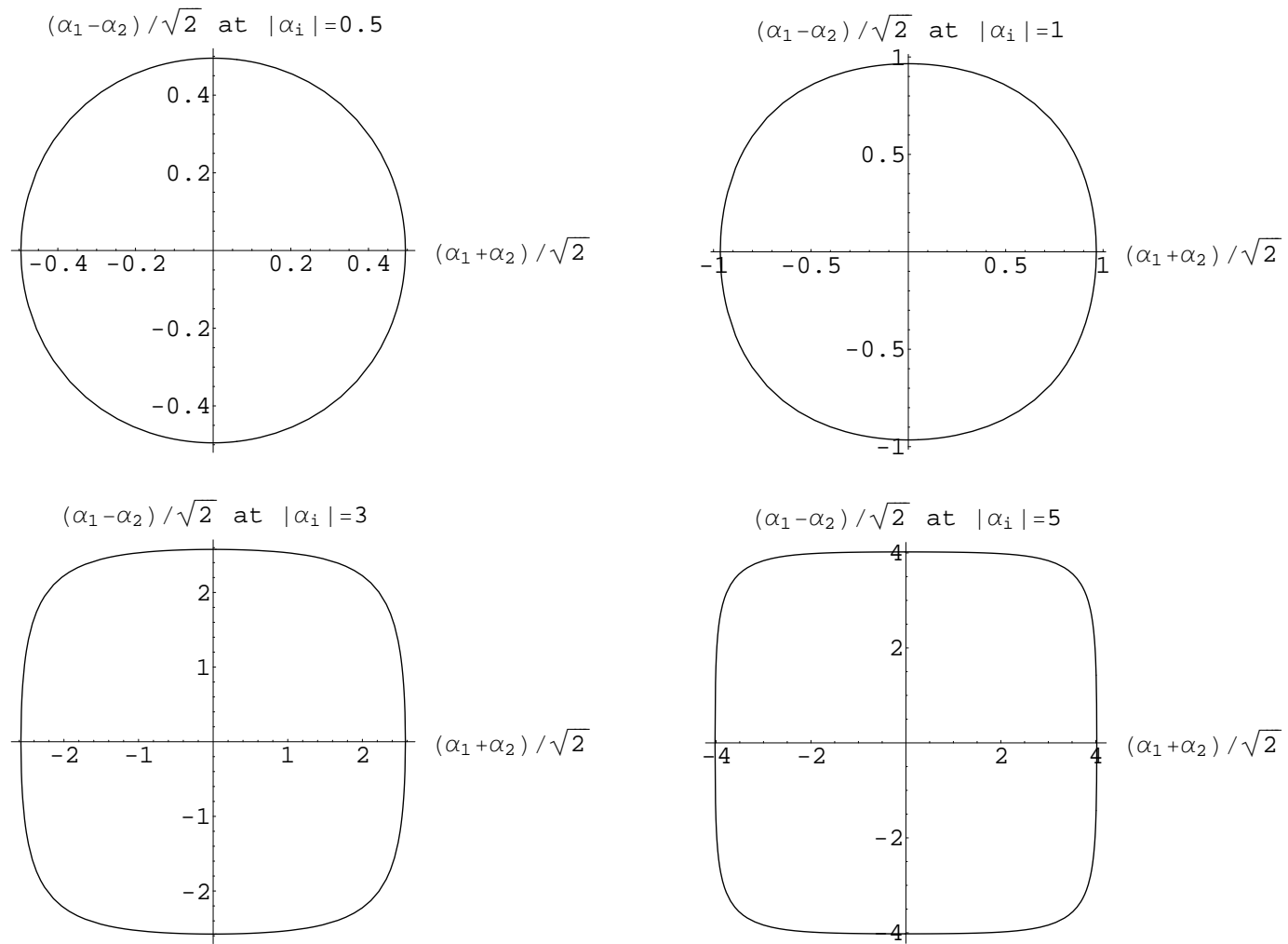

Figure 2. The sections of surfaces $\left|\alpha_{i}\right|=0.5,\left|\alpha_{i}\right|=1,\left|\alpha_{i}\right|=3$ and $\left|\alpha_{i}\right|=5$ by plane $\alpha_{3}=0$, demonstrating the fact that, at $\left|\alpha_{i}\right| \rightarrow \infty$, the section of surface $\left|\alpha_{i}\right|=$ const by the same plane tends to the section of the light front surface shown in Fig. 3.

To illustrate the above described transformation of spheres into a regular rhombic dodecahedron, we shall consider first the behavior of the section of surface $\left|\alpha_{i}\right|=$ const by plane $\alpha_{3}=0$.

The equation that describes the section is

$$
\begin{aligned}
\frac{\alpha_{1}+\alpha_{2}}{\sqrt{2}}= & \pm \frac{1}{\sqrt{2}} \ln \left\{2 \cosh \left|\alpha_{i}\right|-\cosh \sqrt{2}\left(\frac{\alpha_{1}-\alpha_{2}}{\sqrt{2}}\right)\right. \\
& \left.+\left[\left(2 \cosh \left|\alpha_{i}\right|-\cosh \sqrt{2}\left(\frac{\alpha_{1}-\alpha_{2}}{\sqrt{2}}\right)\right)^{2}-1\right]^{1 / 2}\right\},
\end{aligned}
$$

with the variation limits for the argument of function (32) being

$$
\left.\frac{\alpha_{1}-\alpha_{2}}{\sqrt{2}}\right|_{\alpha_{1}+\alpha_{2}=0}=\mp \frac{1}{\sqrt{2}} \ln \left\{2 \cosh \left|\alpha_{i}\right|-1+\left[\left(2 \cosh \left|\alpha_{i}\right|-1\right)^{2}-1\right]^{1 / 2}\right\} \text {. }
$$

Incidentally, it should be noted that, when deriving (32) we not only put $\alpha_{3}=0$ in the initial equation (30), but also introduced new variables $\left(\alpha_{1}+\alpha_{2}\right) / \sqrt{2}$ and $\left(\alpha_{1}-\alpha_{2}\right) / \sqrt{2}$ instead of $\alpha_{1}$ and $\alpha_{2}$. The function (32) is plotted in Fig. 2 at $\left|\alpha_{i}\right|=0.5,\left|\alpha_{i}\right|=1,\left|\alpha_{i}\right|=3$ and $\left|\alpha_{i}\right|=5$. From the plots it is seen how, as $\left|\alpha_{i}\right|$ increases, the circle gets transformed gradually into the square $\beta \alpha \epsilon \delta$ shown in Fig. 3.

Finally, to complete the picture, examine the behavior of the section of surface $\left|\alpha_{i}\right|=$ const by plane $\left(\alpha_{1}+\alpha_{2}\right)=0$. The equation that describes the section is

$$
\frac{\alpha_{1}-\alpha_{2}}{\sqrt{2}}= \pm \frac{1}{\sqrt{2}} \ln \left\{\left(\cosh ^{2} 2 \alpha_{3}+2 \cosh 2\left|\alpha_{i}\right|+1\right)^{1 / 2}-\cosh 2 \alpha_{3}\right.
$$




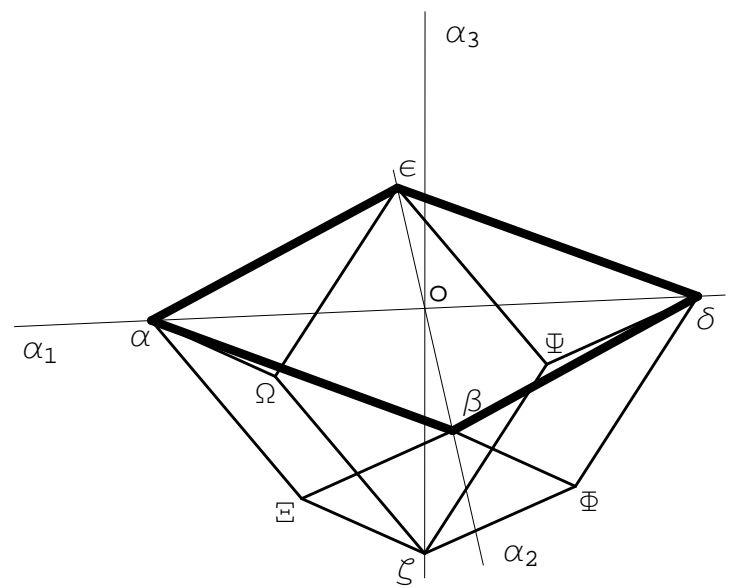

Figure 3. Bold-faced square $\beta \alpha \epsilon \delta$ as section of the light front surface (of the regular rhombic dodecahedron) by plane $\alpha_{3}=0$.

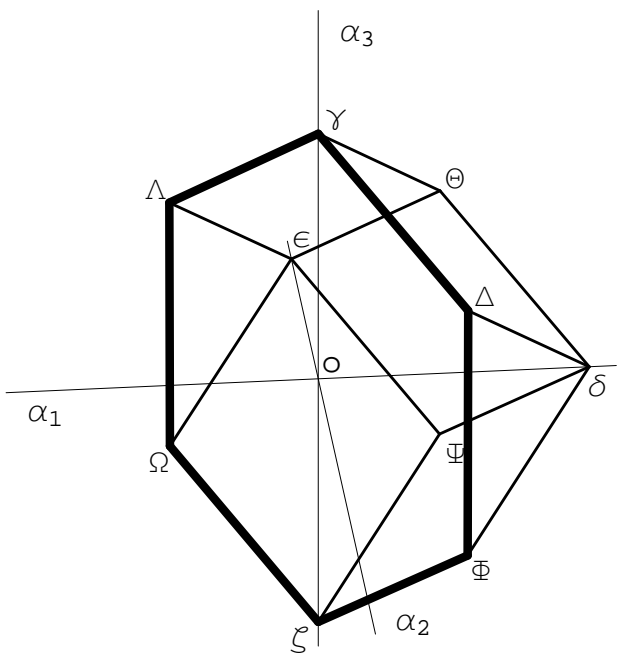

Figure 4. Bold-faced hexagon $\Lambda \gamma \Delta \Phi \zeta \Omega$ as section of the light front surface (of the regular rhombic dodecahedron) by plane $\left(\alpha_{1}+\alpha_{2}\right)=0$.

$$
\left.+\left[\left(\left(\cosh ^{2} 2 \alpha_{3}+2 \cosh 2\left|\alpha_{i}\right|+1\right)^{1 / 2}-\cosh 2 \alpha_{3}\right)^{2}-1\right]^{1 / 2}\right\}
$$

The variation limits for the argument of function (33) are $\left.\alpha_{3}\right|_{\alpha_{1}-\alpha_{2}=0}=\mp\left|\alpha_{i}\right|$.

It should be noted that, when deriving equation (33), we first introduced in (30) $\left(\alpha_{1}+\alpha_{2}\right) / \sqrt{2}$ and $\left(\alpha_{1}-\alpha_{2}\right) / \sqrt{2}$ instead of variables $\alpha_{1}$ and $\alpha_{2}$ and, after that, put $\left(\alpha_{1}+\alpha_{2}\right)=0$. Fig. 5 shows the plots of (33) at $\left|\alpha_{i}\right|=0.5,\left|\alpha_{i}\right|=1,\left|\alpha_{i}\right|=3$ and $\left|\alpha_{i}\right|=5$. From the plots it is seen that, as $\left|\alpha_{i}\right|$ increases, the circle becomes gradually the hexagon $\Lambda \gamma \Delta \Phi \zeta \Omega$ shown in Fig. 4.

\section{A relation between the components of observable 3-velocity and its magnitude in the flat Finslerian event space with entirely broken 3D isotropy}

The Minkowski space domain of causally-related events that correspond to motion of a classical particle is well-known to be bounded by light cone surface. In this case, since the light cone equation in orthogonal Galilean coordinates has the simplest (canonical) form, use of the Einstein 

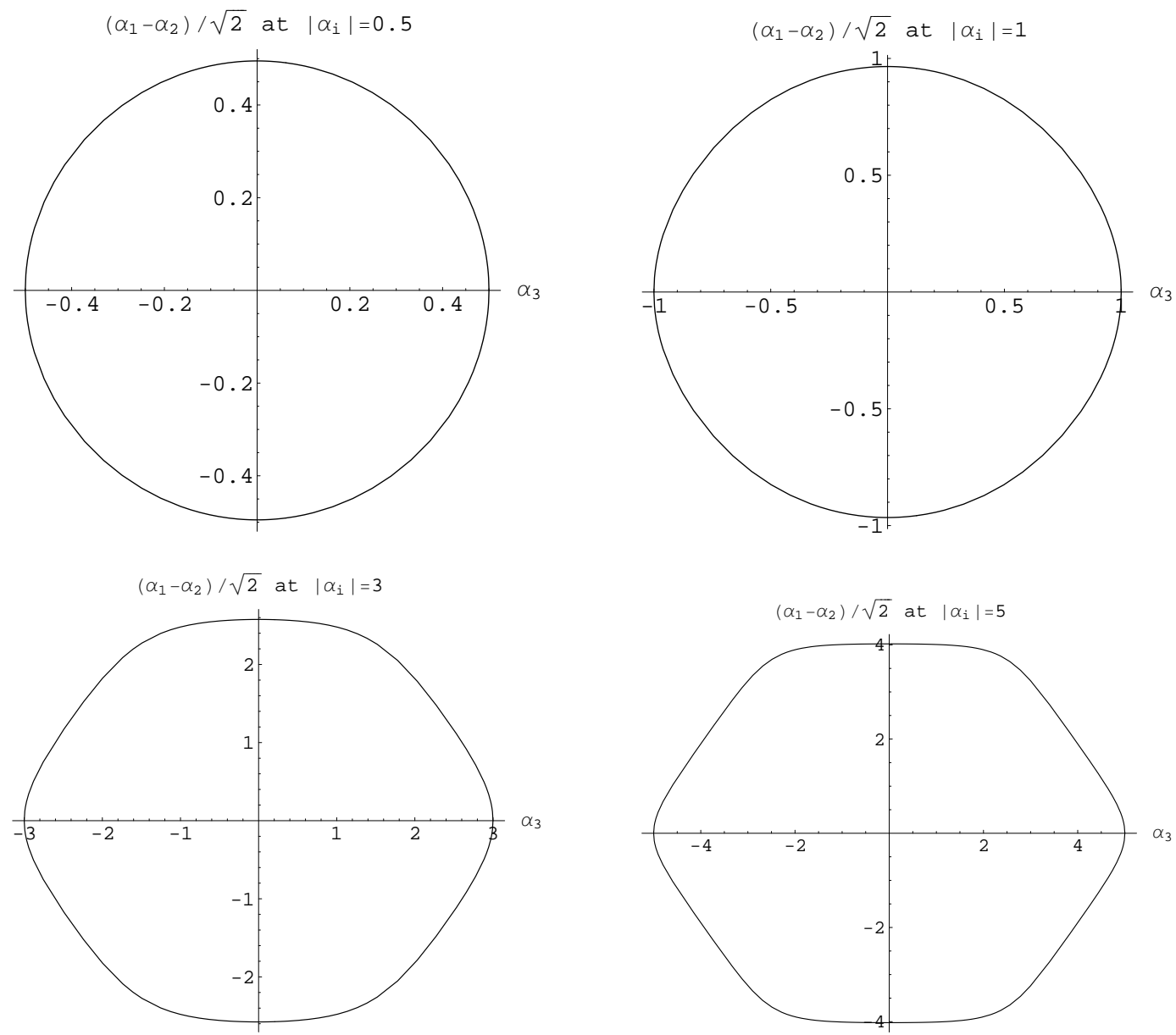

Figure 5. The sections of surfaces $\left|\alpha_{i}\right|=0.5,\left|\alpha_{i}\right|=1,\left|\alpha_{i}\right|=3$ and $\left|\alpha_{i}\right|=5$ by plane $\left(\alpha_{1}+\alpha_{2}\right)=0$ demonstrating the fact that, at $\left|\alpha_{i}\right| \rightarrow \infty$, the section of surface $\left|\alpha_{i}\right|=$ const by the same plane tends to the section of the light front surface shown in Fig. 4.

procedure of exchange of light signals leads to the trivial result, according to which the magnitude of observable 3 -velocity is expressed via the components of the latter as $V^{2}=v_{1}^{2}+v_{2}^{2}+v_{3}^{2}$. Besides, the components $v_{i}$ of observable 3 -velocity coincide in this case with the respective components of coordinate 3 -velocity, so that the range of the physically permissible values of coordinate 3 -velocity of a classical particle is limited by the condition $0 \leq V^{2} \leq 1$, i.e. by a sphere of unit radius. It should be noted that the same situation arises also in the case of Finslerian space with partially broken 3D isotropy (1). However, the range of the physically permissible values of coordinate 3 -velocity $v_{i}$ gets changed significantly when going to entirely anisotropic Finslerian space $(8)$.

To obtain restrictions on the permissible $v_{i}$ values, Hubert Goenner and me [19] made the valid assumption that motion of a particle is in correspondence with such events in space (8), for which not only the condition $d x_{0}>0$, but also the set of inequalities

$$
\begin{aligned}
& d x_{0}-d x_{1}-d x_{2}-d x_{3} \geq 0, \\
& d x_{0}+d x_{1}-d x_{2}+d x_{3} \geq 0, \quad d x_{1}+d x_{2}+d x_{3} \geq 0 \\
& x_{1}+d x_{2}-d x_{3} \geq 0
\end{aligned}
$$

are satisfied, whence the following restrictions on $v_{i}$ were obtained

$$
\begin{aligned}
& 1-v_{1}-v_{2}-v_{3} \geq 0, \\
& 1-v_{1}+v_{2}+v_{3} \geq 0,
\end{aligned}
$$




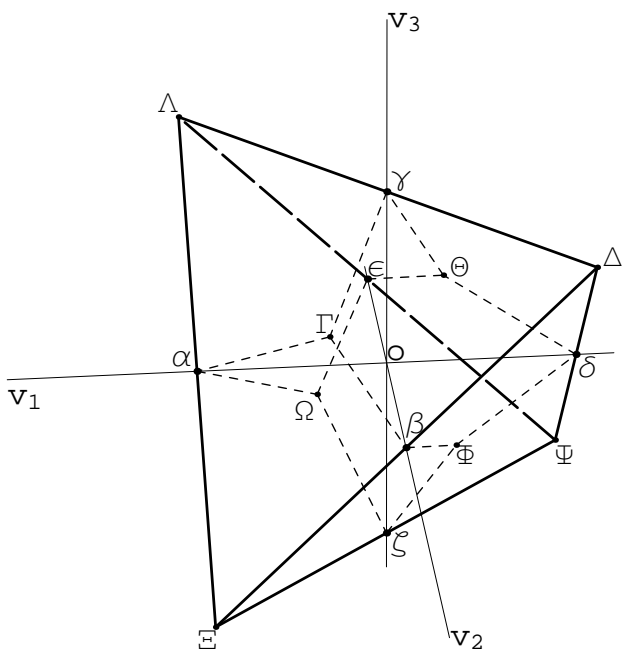

Figure 6. The regular tetrahedron surface in the space of coordinate velocities $v_{i}$ as an image of the light front surface.

$$
\begin{aligned}
& 1+v_{1}-v_{2}+v_{3} \geq 0, \\
& 1+v_{1}+v_{2}-v_{3} \geq 0 .
\end{aligned}
$$

According to (35)-(38), the range of permissible $v_{i}$ values is restricted by the surface of the regular tetrahedron reproduced in Fig. 6. It will be proved below that, in this case the squared magnitude of the respective observable 3-velocity (29) fails to exceed unity, in which case $V^{2}=1$ on the tetrahedron surface only. It is this fact that allows us to state that the domain bounded by the tetrahedron surface serves as the domain of physically permissible coordinate 3 -velocities of a classical particle. It should be noted also that the conditions (35)-(38), which determine that domain, are (at $d x_{0}>0$ ) the necessary and sufficient conditions for relations (34) to be satisfied. We can conclude, therefore, that, from physical viewpoint, the relations (34) single out the domain of causally-related events of event space (8), wherein the world line of any classical particle resides. As to the boundary of that domain, it is determined by the equations that have entered the set (34). Naturally, the boundary can be called the light cone by analogy with the Minkowski space.

It can be readily verified that, when crossing the light cone, we find ourselves in the domain of causally-unrelated events. For that purpose, as clear from the above analysis, it is sufficient to verify that, when crossing the regular tetrahedron surface in the space of coordinate 3velocities $v_{i}$, we enter the domain of supraluminal velocities, i.e. we obtain the values of squared observable 3 -velocity $V^{2}\left(v_{i}\right)$ that exceed unity.

The function $V^{2}\left(v_{i}\right)$ behavior of interest for us can be found out by examining its behavior on the set of all straight lines that pass through the origin $o$ of the Cartesian coordinates $v_{i}$ and, hence, intersect the surface of the regular tetrahedron.

Considered as a typical example will be the behavior of $V^{2}\left(v_{i}\right)$ on a straight line that intersects the tetrahedron surface at points $\Psi$ and $\Gamma$ (see Fig. 6). According to equations (36)-(38), point $\Psi$ (tetrahedron vertex) belongs to faces $\Psi \Xi \Lambda, \Psi \Delta \Xi$ and $\Psi \Lambda \Delta$ and its coordinates are $v_{1}=-1$, $v_{2}=-1, v_{3}=-1$, whereas equation (35) determines the coordinates $v_{1}=1 / 3, v_{2}=1 / 3$, $v_{3}=1 / 3$ of point $\Gamma$, which is located at the center of the face $\Delta \Xi \Lambda$. Therefore, the equations that describe the selected straight line are

$$
v_{1}=t, \quad v_{2}=t, \quad v_{3}=t,
$$

where $t$ is the parameter that characterizes the location of a point on the given line. In particular, $t=-1$ corresponds to point $\Psi, t=0$ to point $o$, and $t=1 / 3$ to point $\Gamma$. Substituting (39) 
in (29), we obtain $V^{2}$ in the form of the following explicit function of running parameter $t$

$$
V^{2}(t)=3 t^{2} /(1-2 t) \text {. }
$$

It is quite obvious now that

i) at the center $o$ of the tetrahedron, i.e. at $t=0, V^{2}(0)=0$;

ii) as $t$ increases, i.e. as we approach the tetrahedron surface, $V^{2}(t)$ increases steadily, so that at $t=1 / 3$ (i.e. at point $\Gamma$ on the tetrahedron surface) $V^{2}(t)$ takes on value $V^{2}(1 / 3)=1$;

iii) as $t$ increases further, i.e. as we move away from the tetrahedron, $V^{2}(t)$ keeps increasing steadily and $\lim _{t \rightarrow 1 / 2} V^{2}(t)=\infty$;

iv) at $t>1 / 2$, we appear in the region where the squared observable 3 -velocity takes on negative values, i.e. $V^{2}(t)<0$;

$\mathrm{v}$ ) as $t$ decreases (from $t=0$ ), i.e. as we move away from point $o$ (where $V^{2}=0$ ) and, hence, as we approach the tetrahedron surface again, $V^{2}(t)$ increases similarly to the case ii) and, at $t=-1$, i.e. at point $\Psi$ on the tetrahedron surface, takes on value $V^{2}(-1)=V^{2}(1 / 3)=1$;

vi) as $t$ decreases further, i.e. as we move away from the tetrahedron again, $V^{2}(t)$ keeps increasing steadily, with $V^{2}(t) \approx-3 t / 2$ at $t \ll-1$.

The method proposed above can be used to study the behavior of function $V^{2}\left(v_{i}\right)$ throughout the space of coordinate velocities $v_{i}$ and (which is of particular importance for quantum theory), to accurately determine the domains in the space of $v_{i}$ where $V^{2}>1$ or $V^{2}<0$. The two domains correspond to propagation of virtual particles only, the first domain corresponding to causally-unrelated (tachyonic) events in space (8). Within the scope of this work, however, we shall limit ourselves to the above analysis. The point is that the behavior of function $V^{2}\left(v_{i}\right)$ along straight line (39) is a good illustration of both the specific features of its behavior throughout the external (with respect to the tetrahedron) domain and the fact that $0 \leq V^{2} \leq 1$ in the domain belonging to the tetrahedron, i.e. just in the domain of $v_{i}$, which is of interest to us in this work.

Return now to Fig. 6. Since the regular tetrahedron surface is an image of the light front surface in the space of $v_{i}$, all the initial light rays and their respective reflected rays divide the tetrahedron into six pairs of mutually conjugate sectors

$$
\begin{array}{ll}
\Gamma \gamma \Delta \beta o \longleftrightarrow \Psi \zeta \Omega \epsilon o, & \Gamma \beta \Xi \alpha o \longleftrightarrow \Psi \epsilon \Theta \delta o, \\
\Gamma \alpha \Lambda \gamma o \longleftrightarrow \Psi \delta \Phi \zeta o, & \Omega \epsilon \Lambda \alpha o \longleftrightarrow \Delta \beta \Phi \delta o, \\
\Omega \alpha \Xi \zeta o \longleftrightarrow \Delta \delta \Theta \gamma o, \quad & \Theta \gamma \Lambda \epsilon o \longleftrightarrow \Xi \zeta \Phi \beta o .
\end{array}
$$

Therefore, the Einstein procedure of exchange of light signals makes it possible to obtain only the respective sectorial components of observable 3-velocity (see [19])

$$
\begin{array}{lll}
v_{\Gamma \gamma \Delta \beta o}=\left(v_{3}+v_{2}\right) /\left(1-v_{1}\right), & v_{\Psi \zeta \Omega \epsilon o}=-\left(v_{3}+v_{2}\right) /\left(1-v_{1}\right), \\
v_{\Gamma \beta \Xi \alpha o}=\left(v_{1}+v_{2}\right) /\left(1-v_{3}\right), & v_{\Psi \epsilon \Theta \delta o}=-\left(v_{1}+v_{2}\right) /\left(1-v_{3}\right), \\
v_{\Gamma \alpha \Lambda \gamma o}=\left(v_{1}+v_{3}\right) /\left(1-v_{2}\right), & v_{\Psi \delta \Phi \zeta o}=-\left(v_{1}+v_{3}\right) /\left(1-v_{2}\right), \\
v_{\Omega \epsilon \Lambda \alpha o}=\left(v_{1}-v_{2}\right) /\left(1+v_{3}\right), & v_{\Delta \beta \Phi \delta o}=-\left(v_{1}-v_{2}\right) /\left(1+v_{3}\right), \\
v_{\Omega \alpha \Xi \zeta o}=\left(v_{1}-v_{3}\right) /\left(1+v_{2}\right), & v_{\Delta \delta \Theta \gamma o}=-\left(v_{1}-v_{3}\right) /\left(1+v_{2}\right), \\
v_{\Theta \gamma \Lambda \epsilon o}=\left(v_{3}-v_{2}\right) /\left(1+v_{1}\right), & v_{\Xi \zeta \Phi \beta o}=-\left(v_{3}-v_{2}\right) /\left(1+v_{1}\right),
\end{array}
$$

where $v_{i}$ are the coordinate 3 -velocity components. As to the relation between the magnitude of observable 3-velocity and its sectorial components, we shall derive it as follows. 
First, using (12) and simplifying the notations in quite an evident way, we represent twelve (actually six) sectorial components of observable 3-velocity as explicit functions of $\alpha_{i}$

$$
\begin{array}{ll}
v_{\Gamma \gamma \Delta \beta o} \equiv v_{3+2}=\tanh \left(\alpha_{3}+\alpha_{2}\right), & v_{\Gamma \beta \Xi \alpha o} \equiv v_{1+2}=\tanh \left(\alpha_{1}+\alpha_{2}\right), \\
v_{\Gamma \alpha \Lambda \gamma o} \equiv v_{1+3}=\tanh \left(\alpha_{1}+\alpha_{3}\right), & v_{\Omega \epsilon \Lambda \alpha o} \equiv v_{1-2}=\tanh \left(\alpha_{1}-\alpha_{2}\right), \\
v_{\Omega \alpha \Xi \zeta o} \equiv v_{1-3}=\tanh \left(\alpha_{1}-\alpha_{3}\right), & v_{\Theta \gamma \Lambda \epsilon o} \equiv v_{3-2}=\tanh \left(\alpha_{3}-\alpha_{2}\right) .
\end{array}
$$

Besides, it is expedient to represent formula (30) as

$$
\begin{aligned}
\tanh ^{2}\left|\alpha_{i}\right|= & 1-8\left[2+\cosh 2\left(\alpha_{1}+\alpha_{2}\right)+\cosh 2\left(\alpha_{1}-\alpha_{2}\right)+\cosh 2\left(\alpha_{3}+\alpha_{2}\right)\right. \\
& \left.+\cosh 2\left(\alpha_{3}-\alpha_{2}\right)+\cosh 2\left(\alpha_{1}+\alpha_{3}\right)+\cosh 2\left(\alpha_{1}-\alpha_{3}\right)\right]^{-1} .
\end{aligned}
$$

Allowing for (18) and using (41) and (40), we obtain

$$
\begin{aligned}
V^{2}= & -8\left[2+\frac{1+v_{1+2}^{2}}{1-v_{1+2}^{2}}+\frac{1+v_{1+3}^{2}}{1-v_{1+3}^{2}}+\frac{1+v_{3+2}^{2}}{1-v_{3+2}^{2}}+\frac{1+v_{1-2}^{2}}{1-v_{1-2}^{2}}\right. \\
& \left.+\frac{1+v_{1-3}^{2}}{1-v_{1-3}^{2}}+\frac{1+v_{3-2}^{2}}{1-v_{3-2}^{2}}\right]^{-1} .
\end{aligned}
$$

According to (42), the square of observable 3-velocity is a symmetric function of its six observable sectorial components (40). It should be noted that these components are constrained by three relations and, hence, are not independent. It can be readily verified that, for example,

$$
v_{1-2}=\frac{v_{1+3}-v_{3+2}}{1-v_{1+3} v_{3+2}}, \quad v_{1-3}=\frac{v_{1+2}-v_{3+2}}{1-v_{1+2} v_{3+2}}, \quad v_{3-2}=\frac{v_{1+3}-v_{1+2}}{1-v_{1+3} v_{1+2}} .
$$

Therefore, any three different observable sectorial components taken from the set (40) serve as one of the possible non-orthogonal basises. For example, having chosen the sectorial components $v_{1+2}, v_{1+3}, v_{3+2}$ to be a particular basis, we can use (42) and (43) to obtain

$$
\begin{aligned}
V^{2}= & 1-\left\{\left(1+v_{1+2} v_{1+3}+v_{1+2} v_{3+2}+v_{1+3} v_{3+2}\right)^{2}\right. \\
& \left.-\left(v_{1+2}+v_{1+3}+v_{3+2}+v_{1+2} v_{1+3} v_{3+2}\right)^{2}\right\} \\
& \times\left\{1-\left(v_{1+2}+v_{1+3}+v_{3+2}-v_{1+2} v_{1+3} v_{3+2}\right)^{2} / 4\right\}^{-1} .
\end{aligned}
$$

If, in particular, $v_{1+3} \neq 0$, while $v_{1+2}=v_{3+2}=0$, then

$$
V^{2}=\frac{3 v_{1+3}^{2}}{4-v_{1+3}^{2}} .
$$

The fact that the square of observable 3-velocity does not coincide (in virtue of (45)) with the square of its sole (non-zero) component $v_{1+3}$ indicates that the given basis is non-orthogonal. On going to, for example, another non-orthogonal basis $v_{1-2}, v_{1+3}, v_{3-2}$ (via (43)) we get $v_{1-2}=v_{1+3}, v_{1+3}=v_{1+3}, v_{3-2}=v_{1+3}$. In this case $v_{1+2}=v_{1-3}=v_{3+2}=0$, so (42) leads to $V^{2}$ expressed again via (45). It should be noted that, since $v_{1+3}$ is an observable sectorial velocity, the maximum value of its square is $v_{1+3}^{2}=1$. In this case, as expected, formula (45) gives $V^{2}=1$.

The situation with observable 3 -velocities in Finslerian event space (8) can be clarified finally by examining the 3 -velocities in non-relativistic limit, i.e.

$$
\left|v_{1+2}\right| \ll 1, \quad\left|v_{1+3}\right| \ll 1, \quad\left|v_{3+2}\right| \ll 1 .
$$


In this case (44) turns into

$$
V^{2}=\left[3\left(v_{1+2}^{2}+v_{1+3}^{2}+v_{3+2}^{2}\right) / 2-v_{1+2} v_{1+3}-v_{1+2} v_{3+2}-v_{1+3} v_{3+2}\right] / 2 .
$$

By its meaning, (47) is a positively determined quadratic form, so it can be reduced to a sum of squares. The explicit form of the appropriate transformation can be found readily. To that end, the first three relations of (40) and relations (12) are sufficient to use with due allowance for (46). As a result we get

$$
v_{1+2}=v_{1}+v_{2}, \quad v_{1+3}=v_{1}+v_{3}, \quad v_{3+2}=v_{3}+v_{2} .
$$

Accordingly, the inverse relations that represent the components $v_{i}$ of coordinate 3 -velocity appear as

$$
\begin{aligned}
& v_{1}=\left(v_{1+3}+v_{1+2}-v_{3+2}\right) / 2, \quad v_{2}=\left(v_{1+2}+v_{3+2}-v_{1+3}\right) / 2, \\
& v_{3}=\left(v_{1+3}+v_{3+2}-v_{1+2}\right) / 2 .
\end{aligned}
$$

On substituting (48) in (47), we see that, in the non-relativistic limit, the squared observable 3velocity equals a sum of squared components of the coordinate 3 -velocity, i.e. $V^{2}=v_{1}{ }^{2}+v_{2}{ }^{2}+v_{3}{ }^{2}$.

\section{Conclusion}

As early as our works [19], where the entirely anisotropic Finslerian event space was first treated, Hubert Goenner and the author encountered the unusual situation in which the Einstein procedure of exchange of light signals failed to properly determine the magnitude of the observable 3 -velocity and was only able to determine the components of the observable 3 -velocity. As a result, the necessity arose that an algorithm be constructed to permit determination of the magnitude of the observable 3 -velocity, thereby formalizing the condition that singles out the domain of causally-related events in space (8). The said events are characterized by the fact that the respective magnitudes of observable 3 -velocities do not exceed the speed of light.

Although constructing the above-mentioned algorithm as realized in the present work was partly heuristic (the identification of functions (27) and (28) to be those exhibiting special transformational properties is meant), the comprehensive analysis of the final formula (29) has shown that it determines the magnitude of observable 3-velocity correctly. Besides, according to (42), formula (29) has led to quite a reasonable relation between the components of observable 3 -velocity and its magnitude. Thus, in addition to the isometry group (10), we have now at our disposal another important tool to study the behavior of fundamental fields in entirely anisotropic Finslerian space (8). The respective studies, as well as the explicit form of the fundamental field equations generalized to meet the requirement of invariance with respect to group (10), will be published elsewhere. As to the present work, to avoid any possible misunderstandings, the following is expedient to note.

It is common knowledge that, in the conventional relativistic quantum field theory, 4-momentum $\left(p_{0}, \boldsymbol{p}\right)$ conjugated canonically to orthogonal Galilean coordinates $\left(x_{0}, \boldsymbol{x}\right)$ is more fundamental than 3-velocity $\boldsymbol{v}$. At the same time, in the case of a free single-particle state with certain 4-momentum $\left|p_{0}, \boldsymbol{p}\right\rangle$, the 3-velocity as a quantum observable has certain value $\boldsymbol{v}=\boldsymbol{p} / p_{0}$ that coincides with classical observable 3 -velocity. In the presence of Lorentz violation, however, the connection between the momentum and the velocity can be highly nontrivial, the fact that was first demonstrated by Don Colladay and Alan Kostelecký in the original SME paper in [2]. Attention should be paid to the fact that a similar effect occurs also within the framework of relativistically invariant Finslerian approach to the Lorentz symmetry violation. In the case of 
flat axially symmetric Finslerian event space (1), for instance, for a free single-particle state with certain 4-momentum $\left|p_{0}, \boldsymbol{p}\right\rangle$, the 3 -velocity as a quantum observable has certain value

$$
\boldsymbol{v}=\frac{(1+r)\left(p_{0}-\boldsymbol{p} \boldsymbol{\nu}\right) \boldsymbol{p}-r\left(p_{0}^{2}-\boldsymbol{p}^{2}\right) \boldsymbol{\nu}}{(1+r)\left(p_{0}-\boldsymbol{p} \boldsymbol{\nu}\right) p_{0}-r\left(p_{0}^{2}-\boldsymbol{p}^{2}\right)}
$$

that coincides with classical observable 3 -velocity in the said event space (see the original paper [4]).

In the case of flat entirely anisotropic Finslerian event space (8) the above notion of 3-velocity involves subtleties associated with the fact that 4-momentum $\left(p_{0}, p_{i}\right)$ is canonically conjugate to nonorthogonal Galilean coordinates $\left(x_{0}, x_{i}\right)$ used to prescribe metric (8). For a free singleparticle state with certain 4-momentum $\left|p_{0}, p_{i}\right\rangle$, as a result, the 3-velocity $v_{i}$ as a quantum observable has the certain value, which is the solution for the set of algebraic equations

$$
a_{q i} v_{i}=b_{q}
$$

where

$$
\begin{aligned}
& a_{11}=a_{12}=\left(p_{0}+p_{3}\right)\left(1+r_{3}\right)-\left(p_{1}+p_{2}\right)\left(r_{1}+r_{2}\right), \\
& a_{13}=b_{1}=\left(p_{1}+p_{2}\right)\left(1+r_{3}\right)-\left(p_{0}+p_{3}\right)\left(r_{2}+r_{3}\right), \\
& a_{21}=-b_{2}=\left(p_{0}-p_{1}\right)\left(r_{2}-r_{3}\right)-\left(p_{2}-p_{3}\right)\left(1-r_{1}\right), \\
& a_{22}=-a_{23}=\left(p_{0}-p_{1}\right)\left(1-r_{1}\right)-\left(p_{2}-p_{3}\right)\left(r_{2}-r_{3}\right), \\
& a_{31}=b_{3}=\left(p_{2}+p_{3}\right)\left(1+r_{1}\right)-\left(p_{0}+p_{1}\right)\left(r_{2}+r_{3}\right), \\
& a_{32}=a_{33}=\left(p_{0}+p_{1}\right)\left(1+r_{1}\right)-\left(p_{2}+p_{3}\right)\left(r_{2}+r_{3}\right) .
\end{aligned}
$$

In the given case, thus, the value of $v_{i}$ taken as a quantum observable coincides with classical coordinate 3 -velocity (see [32]). This implies that relations (29) and (40) should be also used to determine the magnitude and the components of the observable 3 -velocity that would correspond to the free single-particle state with certain 4-momentum $\left|p_{0}, p_{i}\right\rangle$.

It should be emphasized that the single-particle states with certain 4-momentum occur in either of the types of flat anisotropic Finslerian spaces, with pure kinetic part of the 4-momentum being in principle impossible to separate from the latter. This fact indicates that any interaction induced by one or another anisotropy of the homogeneous medium that fills space (1) or space (8) is nonseparable, while the above-mentioned single-particle states are the collective excitations (quasi-particles) that exist in either medium.

The most likely candidate as entirely anisotropic medium that creates anisotropy of space (8) is a three-gluon condensate. A feasibility of forming the three-gluon condensate ensues from work [33] that demonstrates a spontaneous generation of three-gluon gauge invariant effective interaction. Note in the interim that the inert mass tensor for nonrelativistic quasi-particles in entirely anisotropic crystal-like medium is determined by formula (9).

As to axially symmetric anisotropic medium that creates anisotropy of space (1), an axially symmetric fermion-antifermion condensate simulates this sort of crystal-like medium. The condensate appears as a vacuum solution of the Finslerian theory of fermions, whose Lagrangian (7) is constructed proceeding from the requirement of invariance with respect to group $\operatorname{DISIM}_{b}(2)$. After the appropriate shift and expansion, the theory (7) gets quite defined, so the condensate stability problem can be treated within the framework of that theory. This problem is important to solve just because the existing stringent experimental bounds on the space anisotropy magnitude may be indicative of feasible evaporation of the axially symmetric fermion-antifermion condensate, resulting in the present-day very small space anisotropy. If the condensate evaporation assumption proves to be correct, then the question posed in [16], namely, why is the anisotropy so small, may be answered. 
Strictly speaking, the condensate stability problem must be solved in terms of such an extension of the theory (7) that would include couplings to other fields, to gravity field in particular. Although a possibility does exist in principle for the theory (7) to be extended appropriately within the framework of Finslerian approach to the Lorentz symmetry violation, realizing such a plan falls outside the scope of the present work. It should be noted in conclusion, nevertheless, that the first notable attempt of describing the anisotropy-gravity interaction in terms of the Finslerian model for curved space-time was made in [8]. We cannot but mention also work [11], wherein Alan Kostelecký demonstrated that gravity with explicit Lorentz violation could not be described by Riemann (or even Riemann-Cartan) geometry and suggested on his own a Finslerian origin for it instead.

\section{Acknowledgements}

The author is indebted to Boris Arbuzov for informative discussion concerning the feasibility of forming the three-gluon condensate. The author is also thankful to Gary Gibbons for a helpful discussion of the status of the present-day experimental upper bounds on the space anisotropy magnitude and the prospects of the relevant fresh experiments. Finally, the author expresses his gratitude to Dimitri Pavlov and Grigori Garas'ko for the inspiring discussions that stimulated preparing the present work.

Separately, the author would like to thank Hubert Goenner for many-year fruitful collaboration and also the Referees for their valuable remarks that have permitted the paper to be much improved.

This work was supported in part by the Russian Foundation for Basic Research under grant RFBR-07-01-91681-RA_a and by the Non-Commercial Foundation for Finsler Geometry Research.

\section{References}

[1] Kostelecký A., Samuel S., Spontaneous breaking of Lorentz symmetry in string theory, Phys. Rev. D 39 (1989), 683-685.

[2] Colladay D., Kostelecký A., CPT violation and the standard model, Phys. Rev. D 55 (1997), 6760-6774, hep-ph/9703464.

Colladay D., Kostelecký A., Lorentz-violating extension of the standard model, Phys. Rev. D 58 (1998), 116002, 23 pages, hep-ph/9809521.

[3] Kostelecký A. (Editor), CPT and Lorentz symmetry III, Singapore, World Scientific, 2005. Kostelecký A. (Editor), CPT and Lorentz symmetry IV, Singapore, World Scientific, 2008.

[4] Bogoslovsky G.Yu., A special relativistic theory of the locally anisotropic space-time, Nuovo Cimento B 40 (1977), 99-134, Erratum, Nuovo Cimento B 43 (1978), 377-378.

[5] Tavakol R., van den Bergh N., Finsler spaces and the underlying geometry of space-time, Phys. Lett. A 112 (1985), 23-25.

[6] Tavakol R., van den Bergh N., Viability criteria for the theories of gravity and Finsler spaces, Gen. Relativity Gravitation 18 (1986), 849-859.

[7] Bogoslovsky G.Yu., Theory of locally anisotropic space-time, Moscow, Moscow Univ. Press, 1992.

[8] Bogoslovsky G.Yu., Finsler model of space-time, Phys. Part. Nucl. 24 (1993), 354-379.

Bogoslovsky G.Yu., A viable model of locally anisotropic space-time and the Finslerian generalization of the relativity theory, Fortschr. Phys. 42 (1994), 143-193.

[9] Bogoslovsky G.Yu., Goenner H.F., Concerning the generalized Lorentz symmetry and the generalization of the Dirac equation, Phys. Lett. A 323 (2004), 40-47, hep-th/0402172.

[10] Bogoslovsky G.Yu., Subgroups of the group of generalized Lorentz transformations and their geometric invariants, SIGMA 1 (2005), 017, 9 pages, math-ph/0511077.

Bogoslovsky G.Yu., Lorentz symmetry violation without violation of relativistic symmetry, Phys. Lett. A 350 (2006), 5-10, hep-th/0511151. 
[11] Kostelecký A., Gravity, Lorentz violation, and the Standard Model, Phys. Rev. D 69 (2004), 105009, 20 pages, hep-th/0312310.

[12] Bailey Q.G., Kostelecký A., Signals for Lorentz violation in post-Newtonian gravity, Phys. Rev. D 74 (2006), 045001, 46 pages, gr-qc/0603030.

[13] Girelly F., Liberati S., Sindoni L., Planck-scale modified dispersion relations and Finsler geometry, Phys. Rev. D 75 (2007), 064015, 9 pages, gr-qc/0611024.

[14] Ghosh S., Pal P., Deformed special relativity and deformed symmetries in a canonical framework, Phys. Rev. D 75 (2007), 105021, 11 pages, hep-th/0702159.

[15] Bogoslovsky G.Yu., Some physical displays of the space anisotropy relevant to the feasibility of its being detected at a laboratory, arXiv:0706.2621.

[16] Gibbons G.W., Gomis Joaquim, Pope C.N., General very special relativity is Finsler geometry, Phys. Rev. D 76 (2007), 081701(R), 5 pages, arXiv:0707.2174.

Cohen A.G., Glashow S.L., Very special relativity, Phys. Rev. Lett. 97 (2006), 021601, 3 pages, hep-ph/0601236.

[17] Mavromatos N.E., Lorentz invariance violation from string theory, arXiv:0708.2250.

[18] Sindoni L., The Higgs mechanism in Finsler spacetimes, arXiv:0712.3518.

[19] Bogoslovsky G.Yu., Goenner H.F., On the possibility of phase transitions in the geometric structure of space-time, Phys. Lett. A 244 (1998), 222-228, gr-qc/9804082.

Bogoslovsky G.Yu., Goenner H.F., Finslerian spaces possessing local relativistic symmetry, Gen. Relativity Gravitation 31 (1999), 1565-1603, gr-qc/9904081.

[20] Bogoslovsky G.Yu., On a special relativistic theory of anisotropic space-time, Dokl. Akad. Nauk SSSR 213 (1973), 1055-1058.

[21] Patera J., Winternitz P., Zassenhaus H., Continuous subgroups of the fundamental groups of physics. II. The similitude group, J. Math. Phys. 16 (1975), 1615-1624.

[22] Winternitz P., Friš I., Invariant expansions of relativistic amplitudes and subgroups of the proper Lorentz group, Yadern. Fiz. 1 (1965), 889-901.

[23] Bogoslovsky G.Yu., The proper time, spatial distances and clock synchronization in the locally anisotropic space-time, JINR Communication E2-82-779, Dubna, JINR, 1982.

[24] Bogoslovsky G.Yu., The relativistic inert mass tensor, Vestn. Mosk. Univ. Ser. Fiz. Astron. 24 (1983), no. 1, 70-71.

[25] Bogoslovsky G.Yu., On the local anisotropy of space-time, inertia and force fields, Nuovo Cimento B $\mathbf{7 7}$ (1983), 181-190.

[26] Bogoslovsky G.Yu., A generalized Klein-Gordon equation and Mach's principle, Vestn. Mosk. Univ. Ser. Fiz. Astron. 24 (1983), no. 3, 59-61.

[27] Bogoslovsky G.Yu., Goenner H.F., On the generalization of the fundamental field equations for locally anisotropic space-time, in Proceedings of XXIV International Workshop "Fundamental Problems of High Energy Physics and Field Theory" (June 27-29, 2001, Protvino, Russia), Editor V.A. Petrov, Protvino, Insitute for High Energy Physics, 2001, 113-125, http://dbserv.ihep.su/ pubs/tconf01/c2-5.htm.

[28] Berwald L., Projective Krümmung allgemeiner affiner Räume und Finslersche Räume skalarer Krümmung, Ann. Math. 48 (1947), 755-781 (und die Literaturhinweise darin).

[29] Moór A., Ergänzung, Acta. Math. 91 (1954), 187-188.

[30] Weyl H., Gravitation und Elektrizität, Sitzber. preuss Akad. Wiss., Physik-math. Kl., 1918, 465-480. Weyl H., Eine neue Erweiterung der Relativitätstheorie, Ann. Phys. 59 (1919), 101-133.

[31] Bogoslovsky G.Yu., From the Weyl theory to a theory of locally anisotropic space-time, Classical Quantum Gravity 9 (1992), 569-575.

[32] Bogoslovsky G.Yu., 4-momentum of a particle and the mass shell equation in the entirely anisotropic spacetime, in Space-Time Structure (Algebra and Geometry), Editors D.G. Pavlov, Gh. Atanasiu and V. Balan, Moscow, Lilia Print, 2007, 156-173.

[33] Arbuzov B.A., Infrared non-perturbative QCD running coupling from Bogolubov approach, Phys. Lett. B 656 (2007), 67-73, hep-ph/0703237. 